\title{
ON A CLASS OF NONLINEAR DIFFERENCE EQUATIONS IN THE COMPLEX DOMAIN
}

\author{
BY \\ WALTER STRODT
}

\section{Part I. Introduction.}

1. Notation. Throughout the paper $\beta$ will represent a fixed positive number not exceeding $\pi / 2$.

2. Notation. If $\gamma$ is any positive number not exceeding $\pi / 2$, and if $\xi$ is any real number, then by $\mathcal{S}_{\gamma}(\xi)$ is meant the sector $\{x ; x \neq \xi$, $|\arg (x-\xi)|<\gamma\}\left({ }^{1}\right)$.

3. Definition. A function $\phi(x)$ will be called admissible if it has the following two properties:

(3.1) for every positive number $\gamma$ less than $\beta$ there is a positive number $\xi$ such that $\phi(x)$ is analytic and bounded in $\oint_{\gamma}(\xi)$, and axis.

(3.2) $\phi(x)$ approaches a limit as $x$ becomes infinite on the positive real

4. Introductory remarks on the nature of the problem. The difference equations to be studied in this paper are algebraic in the unknown function and its translations, and have coefficients which are admissible functions of $x$. Under the hypothesis that the constants approached by the coefficients (as $x$ becomes infinite on the positive real axis) do not satisfy certain equations, a complete description is given of the totality $\mathcal{T}$ of all those solutions of the difference equations, which, like the coefficients, are admissible functions of $x$.

The zeros of a certain auxiliary polynomial being $\sigma_{1}, \cdots, \sigma_{c}$, it is shown that $\mathcal{T}$ is the union of finitely many disjoint subsets $\tau_{1}, \cdots, \tau_{c}$, all the solutions in $\mathcal{T}_{k}$ tending to $\sigma_{k}$ on the positive real axis $(k=1,2, \cdots, c)$. Each of the sets $\mathcal{T}_{k}$ is a finite-parameter family of functions, the number of parameters, $q_{k}$, being the number of zeros of a certain auxiliary exponential polynomial which lie in a certain sector of the complex plane. It may be remarked that $\mathcal{T}$ is readily seen to be identical with the set of all those solutions which satisfy only the single condition (3.1).

A precise statement of the result is given in Theorem 2 of $\$ 42$, below.

The method of attack is a method of successive approximations, each approximation being obtained from the preceding by means of the solving of a linear nonhomogeneous difference equation with constant coefficients. The formula which is used to solve this linear difference equation is a slight modification of a formula obtained by the author in an earlier paper $\left(^{2}\right)$.

Presented to the Society, April 30, 1949; received by the editors April 20, 1949.

( $\left.{ }^{1}\right)$ Throughout this paper it is understood that for every nonzero $x$ the number arg $x$ is chosen to satisfy the inequalities $-\pi<\arg x \leqq \pi$.

(2) Linear difference equations and exponential polynomials, Trans. Amer. Math. Soc. vol. 64 (1948) pp. 439-466; this paper will hereinafter be designated as [L]. 
The concept of a finite-parameter family of functions is made precise by the introduction of a suitable metric for the function-space $\mathcal{T}_{k}$, a metric in terms of which $\tau_{k}$ is a manifold $\left(^{3}\right)$ of $q_{k}$ complex dimensions.

The results of this paper are in a certain sense continuations of results which the author obtained in an earlier paper $\left(^{4}\right)$. Roughly speaking, the hypothesis of $[\mathrm{P}]$ required that a certain auxiliary exponential polynomial never vanish on the nonpositive real axis; the present paper permits this exponential polynomial to vanish at any points except at 0 and at $-\infty$. However, in the present paper the coefficients and solutions are required to be "admissible," instead of "almost constant" as in $[\mathrm{P}]$; this is a much weaker restriction.

A step in the proof of Theorem 2 is Theorem 1 , in $\$ 41$, below. This describes, in an explicit manner, the totality of all admissible solutions of the general linear difference equation $\sum_{j=1}^{n} A_{j} y\left(x+\omega_{j}\right)=\phi(x)$ with constant $A_{j}$ and admissible $\phi(x)$, under the assumptions $A_{1} \neq 0, \sum_{j=1}^{n} A_{j} \neq 0$.

Part II. Preliminaries on exponential polynomials.

5. Notations. If $\gamma$ is any positive number not exceeding $\beta$, then by the symbol $\mathcal{N}_{\gamma}$ will be meant the complement of the sector $\{x ; x \neq 0 ;|\arg x|<\gamma$ $+\pi / 2\}$. If in addition $\xi$ is any real number, then by $\mathcal{C}_{\gamma}(\xi)$ will be mean the class of all functions which are analytic and bounded in the sector $\mathcal{S}_{\gamma}(\xi)$.

6. Lемма . Let $n$ be a positive number greater than unity. Let $\omega_{1}=0$, and let $\omega_{2}, \cdots, \omega_{n}$ be any distinct complex numbers in $\mathcal{S}_{\beta}(0)$. Let $A_{1}, \cdots, A_{n}$ be any complex numbers such that $A_{1} \neq 0$, and such that $\sum_{j=1}^{n} A_{j} \neq 0$. Let $f(z)$ be the exponential polynomial $\sum_{j-1}^{n} A_{j} e^{\omega_{j} z}$. Let $\zeta_{1}, \zeta_{2}, \cdots$ be the distinct zeros of $f(z)$. Let $\delta$ be any positive number. Let $Z(\delta)$ be the set of all points $x$ such that the distance of $x$ from the set $\left\{\zeta_{1}, \zeta_{2}, \cdots\right\}$ is less than $\delta$. Let $G_{1}(\delta), G_{2}(\delta), \cdots$ be the connected components of $\mathcal{Z}(\delta)$. Then there are positive numbers $\delta_{0}$ and $C$, and $a$ non-negative integer $p$, and a sequence of real numbers $\gamma_{p+1}, \gamma_{p+2}, \cdots$ in the open interval $(-\beta, \beta)$, there being only finitely many distinct members in the set $\left\{\gamma_{p+1}, \gamma_{p+2}, \cdots\right\}$, such that if $\delta<\delta_{0}$, and if the $\zeta_{s}$ and the $G_{s}(\delta)$ are suitably numbered, then:

(a) $\zeta_{1}, \cdots, \zeta_{p}$ are precisely the zeros of $f(z)$ in $\mathcal{N}_{\beta}$;

(b) There are at most $n-1$ distinct points $\zeta_{s}$ in $G_{8}(\delta)(s=p+1, p+2, \cdots)$;

(c) $\zeta_{s}$ is the only zero of $f(z)$ in $G_{8}(\delta)(s=1,2, \cdots, p)$;

(d) The positively sensed complete boundary $\mathcal{F}_{8}(\delta)$ of $G_{s}(\delta) \quad(s=p+1$, $p+2, \cdots)$ is of length not more than $2 \pi(n-1) \delta$.

(e) There exists a positive number $\lambda(\delta)$ such that $|f(z)|>\lambda(\delta)$ for all $z$ on $\mathcal{F}_{s}(\delta)(s=p+1, p+2, \cdots)$;

(3) That is, a connected separable metric space which is locally homeomorphic with $g_{k}$-dimensional complex Euclidean space. $\mathcal{T}_{k}$ can be easily seen to be a complete space as well.

(4) Principal solutions of difference equations, Amer. J. Math. vol. 69 (1947) pp. 717-757; this paper will hereinaf ter be designated as $[\mathrm{P}]$. 
(f) $\Re(z) \cos \gamma_{s}-\Im(z) \sin \gamma_{s}>C s$ if $z$ is on $\mathcal{F}_{s}(\delta)$ and $s>p\left({ }^{5}\right)$.

(g) $|z|>C s$ if $z$ is on $\mathcal{F}_{\mathrm{s}}(\delta)(s=1,2, \cdots)$.

Proof. This lemma follows immediately from [L: Theorem 1c, 1d, 1e, Lemma 4a, 4d, and Lemma 1a]. The condition that the $A_{j}$ be all different from zero [L: notation (7), p. 440] is not necessarily fulfilled under the hypotheses of the present lemma, but it is readily seen that the $A_{j}$ which are zero can be ignored in applying the results of [L]. Also, it may be noted that the number $N$ defined in [L: notation 20, p. 441] is necessarily less than or equal to $n$. Finally, it may be observed that the set $\mathcal{N}(0, \beta)$ defined in [L: notation 26, p. 441 ] is precisely the set $\mathcal{N}_{\beta}$ of the present paper.

7. Notation. We make a particular choice of the numbers $\delta_{0}, \delta, C, \gamma_{p+1}$, $\gamma_{p+2}, \cdots, \lambda(\delta)$ satisfying the conditions of Lemma 1 , and shall retain this choice throughout this paper. With this in mind, we shall not exhibit in our subsequent notation the fact that various functions may depend upon this choice, as well as upon variables appearing explicitly as arguments.

8. Notation. Let $\beta_{1}=1$.u.b. $\left\{\left|\arg \omega_{j}\right|,\left|\gamma_{m}\right|,\left|\arg \zeta_{m}\right|-\pi / 2 ; j=2, \cdots, n\right.$; $m=p+1, p+2, \cdots\}$.

9. LEMMA 2. $\beta_{1}<\beta$.

Proof. Since the numbers arg $\omega_{j}$ and $\gamma_{m}$ are finite in number and lie in the open interval $(-\beta, \beta)$, it suffices to prove that l.u.b. $\left\{\left|\arg \zeta_{m}\right| ; m=p+1\right.$, $p+2, \cdots\}<\beta+\pi / 2$. By [L: Lemma 4e], with $M_{1}=0$, there is a positive number $\theta$ less than $\beta$ such that $\zeta_{1}, \cdots, \zeta_{p}$ are precisely all the zeros of $f(z)$ in $\mathcal{N}_{\theta}$. This implies that $\left|\arg \zeta_{m}\right|<\theta+\pi / 2(m=p+1, p+2, \cdots)$. Hence 1.u.b. $\left\{\left|\arg \zeta_{m}\right| ; m=p+1, p+2, \cdots\right\} \leqq \theta+\pi / 2<\beta+\pi / 2$.

10. Notation. It will be convenient to choose, once for all, a positive number $\alpha$ greater than $\beta_{1}$, and less than $\beta$. This choice we make now. In the interests of definiteness, we define $\alpha=\left(\beta_{1}+\beta\right) / 2$.

11. Lemma 3. The zeros of $f(z)$ which lie in $\mathcal{N}_{\alpha}$ are precisely $\zeta_{1}, \cdots, \zeta_{p}$.

Proof. This follows at once from the definitions of $\alpha$ and $\mathcal{N}_{\alpha}$.

Part III. Preliminaries on admissible functions.

12. Notation. If $\phi(x)$ is any function which is in $\mathcal{C}_{\gamma}(\xi)$, then by $B_{\gamma \xi}[\phi(x)]$ is meant l.u.b. $\left\{|\phi(x)| ; x \in \mathcal{S}_{\gamma}(\xi)\right\}$. If $\phi(x)$ is any function which is analytic and bounded on the half-line $\{x ; x>\xi\}$, then by $B_{0 \xi}[\phi(x)]$ is meant l.u.b. $\{|\phi(x)| ; x>\xi\}$.

13. Definition. A function $\phi(x)$ will be called remotely bounded if it satisfies condition (3.1).

14. Notation. If $g\left(x, t_{1}, \cdots, t_{m}\right)$ is a function of the indicated arguments which for a fixed set of values of $t_{1}, \cdots, t_{m}$ tends to a limit as $x$ becomes infinite on the positive real axis, then by $g\left(+\infty, t_{1}, \cdots, t_{m}\right)$ is meant that limit.

(b) $\Re$ and $\Im$ will mean real and imaginary part, respectively. 
15. Lemma 4. If $g(x)$ is admissible, then for every positive $\gamma$ less than $\beta$ and for every positive $\epsilon$ there is a positive $\xi$, depending upon $\gamma$ and $\epsilon$, such that $B_{\gamma \xi}[g(x)-g(+\infty)]<\epsilon$.

Proof. This lemma follows readily from a classic theorem of Lindelöf $\left({ }^{6}\right)$.

16. LEMMA 5. If $g(x)$ is admissible, and if $\gamma<\beta$, and if $\xi$ is sufficiently large so that $g(x)$ is in $C_{\gamma}(\xi)$, then $g(x)-g(+\infty)$ tends to zero as $x$ becomes infinite in the sector $\mathcal{S}_{\gamma}(\xi)$.

Proof. Let $\theta=(\beta+\gamma) / 2$. If $R=|x-\xi|$, and $x$ is in $\mathcal{S}_{\gamma}(\xi)$, then $x \in \mathcal{S}_{\theta}(X)$, where $X=\xi+R \sin (\theta-\gamma) \csc \theta$. Since $X$ becomes infinite with $R$, and since $\theta<\beta$, the lemma now follows from Lemma 4 .

17. Lemma 6. If $g(x)$ is admissible, and if $\gamma<\beta$, and if $g(x)$ is in $C_{\gamma}(\xi)$, then either $g(x) \equiv g(+\infty)$, or else $B_{\gamma X}[g(x)-g(+\infty)]$ decreases monotonically (in the strict sense) as $X$ increases $(X \geqq \xi)$.

Proof. Let $\xi \leqq X_{1}<X_{2}$. Suppose $g(x) \not \equiv g(+\infty)$. Then $B_{\gamma X_{2}}[g(x)-g(+\infty)]$ $>0$. Also, by Lemma $5, g(x)-g(+\infty)$ tends to zero as $x$ becomes infinite $\mathcal{S}_{\gamma}\left(X_{2}\right)$. This implies that there is a point $x_{0}$ in the closed envelope of $\mathcal{S}_{\gamma}\left(X_{2}\right)$ such that $\left|g\left(x_{0}\right)-g(+\infty)\right|=B_{\gamma X_{2}}[g(x)-g(+\infty)]$. Hence, since $g(x)$ is not a constant (by virtue of the assumption $g(x) \not \equiv g(+\infty)$ ), there is a point $x$ in every neighborhood of $x_{0}$ at which $|g(x)-g(+\infty)|>B_{\gamma X_{2}}[g(x)-g(+\infty)]$. This implies that $B_{\gamma X_{1}}[g(x)-g(+\infty)]>B_{\gamma X_{2}}[g(x)-g(+\infty)]$.

18. Notation. Throughout this paper the symbol $D$ will be used to represent the operation of differentiation with respect to $x . D^{k} \phi\left(x_{0}\right)$ will mean, as usual, the $k$ th derivative of $\phi(x)$, evaluated at $x=x_{0}$.

19. Lемма 7. If $g(x)$ is admissible, then $D g(x)$ is admissible.

Proof. This is an obvious consequence of the Cauchy integral formula for the first derivative of $g(x)$.

20. LEMMA 8. If $j$ is any non-negative integer, and if $\zeta_{1}, \cdots, \zeta_{p}$ are the numbers of Lemma $1 \mathrm{a}$, then $x^{j} e^{\zeta_{s} x}$ is admissible $(s=1,2, \cdots, p)$, and its limit at $+\infty$ is zero. Moreover, for every positive number $\gamma$ less than $\beta$ there is a positive number $G_{j s}(\gamma)$ such that $B_{\gamma 0}\left[x^{i} e^{\zeta_{s} x}\right]<G_{j s}(\gamma)$.

Proof. Since $\zeta_{s}$ is not zero (by virtue of the condition $\sum_{j=1}^{n} A_{j} \neq 0$ ), and since it is in $\mathcal{N}_{\beta}$, we have $\Re\left(\zeta_{8}\right) \cos \gamma+\left|\Im\left(\zeta_{8}\right)\right| \sin \gamma<0$. But if $x$ is in $\mathcal{S}_{\gamma}(0)$,

(8) Cf., for example, Nevanlinna, Eindeutige analytische Funktionen, Berlin, 1936, p. 44. The hypotheses of Lemma 4 imply information adequate for the application of the cited theorem, if transformations $\log z=(\pi / \theta) \log (x-X)$ and $\log z=(-\pi / \theta) \log (x-X)$ are made, with $\theta=(\beta+\gamma) / 2$ and with $X$ sufficiently large so that $g(x)$ is bounded in the sector $|\arg (x-X)|$ $<\theta$. 
then $\Re\left(\zeta_{s} x\right) \leqq|x|\left[\Re\left(\zeta_{s}\right) \cos \gamma+\left|\Im\left(\zeta_{s}\right)\right| \sin \gamma\right]$. From this the lemma follows immediately.

Part IV. Preliminaries on certain linear difference operators and equations.

21. REMARK. The formulas used in this part are motivated by [L: equations 42-45].

22. Notations. Using the notations of $\S 6$, let $F(z)=1 / f(z)$, and let $z_{m}=e^{i \gamma m}$ $(m=p+1, p+2, \cdots)$.

23. Definition. Using the notations of $\$ \$ 6$ and 22 , and letting $\xi$ be any real non-negative number, let the operators $L_{\xi_{s}}(s=1,2, \cdots, p)$ and the operators $K_{m}(m=p+1, p+2, \cdots)$ be defined by the following equations, which are to be valid for every function $\phi(x)$ for which they are meaningful:

$$
L_{\xi_{8}} \phi(x)=\int_{\xi}^{x}(2 \pi i)^{-1} \int_{\mathcal{F}_{s}(\delta)} e^{T(x-u)} \phi(u) F(T) d T d u
$$

and

$$
K_{m} \phi(x)=(2 \pi i)^{-1} \int_{\mathcal{F}_{m}(\delta)} F(T) T^{-1} \int_{0}^{\infty} e^{-\rho z_{m} T} D \phi\left(x+\rho z_{m}\right) z_{m} d \rho d T
$$

where the contour of integration for $u$ in (23.1) is the straight line segment $[\xi, \Re(x)]$ followed by the straight line segment $[\Re(x), x]$.

24. Notation. Let $j_{8}$ be the order of multiplicity of $\zeta_{8}$ as a zero of $f(z)$, and let $P_{s}(z)=\sum_{j=1}^{j_{s}} B_{s j}\left(z-\zeta_{s}\right)^{-i}$ be the principal part of $F(z)$ at $\zeta_{s}(s=1,2, \cdots, p)$.

25. Lemma 9. Let $\gamma$ be a positive number less than $\beta$. Let $\xi$ be any non-negative number, and let $\phi(x)$ be any function which is in $\mathcal{C}_{\gamma}(\xi)$. Then

(a) $L_{\xi s} \phi(x)$ is in $C_{\gamma}(\xi)(s=1,2, \cdots, p)$, and moreover,

(b) There exists a positive number $V_{s}(\gamma)$ independent of $\phi(x)$, and of $\xi$, such that $B_{\gamma \xi}\left[L_{\xi} \phi(x)\right] \leqq V_{s}(\gamma) B_{\gamma \xi}[\phi(x)](s=1,2, \cdots, p)$, and, finally,

(c) There exist functions $\eta_{s}(X, \gamma)$, defined for positive $X$, for $s \in(1,2, \cdots, p)$, and for positive $\gamma$ inferior to $\beta$, and functions $U_{1 s}(\gamma), U_{2 s}(\gamma)$, defined for positive $\gamma$ inferior to $\beta$, such that $\eta_{s}(X, \gamma)$ tends to zero as $X$ becomes infinite, and such that if $\xi, X_{1}, X_{2}$ is any triple of real numbers satisfying the relations $0 \leqq \xi<X_{1}<X_{2}$, and if $g(x)$ is any function which is analytic and bounded on the half-line $\{x ; x>\xi\}$, and which is in $C_{\gamma}\left(X_{2}\right)$, then the functions $L_{\xi s} g(x)$ are in $\mathcal{C}_{\gamma}\left(X_{2}\right)$, and

$$
\begin{aligned}
B_{\gamma X_{2}}\left[L_{\xi s} g(x)\right] \leqq & \eta_{s}\left(X_{2}-X_{1}, \gamma\right) B_{0 \xi}[g(x)]+U_{1 s}(\gamma) B_{0 X_{1}}[g(x)] \\
& +U_{2 s}(\gamma) B_{\gamma X_{2}}[g(x)] .
\end{aligned}
$$

Proof. Let $-K(\gamma)=\max \left\{\Re\left(\zeta_{s}\right) \cos \gamma+\left|\Im\left(\zeta_{s}\right)\right| \sin \gamma ; s=1,2, \cdots, p\right\}$. Since $\zeta_{1}, \cdots, \zeta_{p}$ are nonzero complex numbers belonging to $\mathcal{N}_{\beta}$, it is evident that $K(\gamma)>0$. 
Now let $\phi(x)$ be any function which is in $C_{\gamma}(\xi)$.

When $x$ is in $\mathcal{S}_{\gamma}(\xi)$, it is permissible, in the calculation of $L_{\xi} \phi(x)$, to replace the polygonal contour $[\xi, \Re(x)]+[\Re(x), x]$ by the straight line contour $[\xi, x]$. Let this replacement be made. As an immediate consequence of the calculus of residues we have

$$
L_{\xi_{s}} \phi(x)=\sum_{j=1}^{j_{s}} B_{s j}[(j-1) !]^{-1} I_{s j}(x)
$$

where $I_{s j}(x)=\int_{\xi}^{x} \phi(u) e^{\zeta_{s}(x-u)}(x-u)^{j-1} d u$. Let $x=\xi+R e^{i \theta}$, with $R>0$ and with $|\theta|<\gamma$. Then $u=\xi+\rho e^{i \theta}$, with $0 \leqq \rho \leqq R$. Hence $I_{s j}(x)=\int_{0}^{R} \phi\left(\xi+\rho e^{i \theta}\right)$ $\exp \left[\zeta_{s}(R-\rho) e^{i \theta}\right](R-\rho)^{j-1} e^{i j \theta} d \rho$. Now $\Re\left[\zeta_{s} e^{i \theta}\right] \leqq-K(\gamma)$. Hence $\left|I_{s j}(x)\right|$ $\leqq B_{\gamma \xi}[\phi(x)] \cdot \int_{0}^{\infty} e^{-K(\gamma) t} t^{j-1} d t=B_{\gamma \xi}[\phi(x)][K(\gamma)]^{-j}(j-1) !$. Hence $B_{\gamma \xi}\left[L_{\xi 8} \phi(x)\right]$ $\leqq V_{8}(\gamma) B_{\gamma \xi}[\phi(x)]$, where $V_{8}(\gamma)=\sum_{j=1}^{j_{s}}\left|B_{s j}\right|[K(\gamma)]^{-j}$. This establishes (a) and (b).

Now let $\xi, X_{1}, X_{2}$ be any real numbers such that $0 \leqq \xi<X_{1}<X_{2}$. Let $\gamma$ be any positive number less than $\beta$. Let $g(x)$ be any function which is analytic and bounded on the half-line $\{x ; x>\xi\}$, and which is in $\mathcal{C}_{\gamma}\left(X_{2}\right)$.

Let $-K(0)=\max \left\{R\left(\zeta_{s}\right) ; s=1,2, \cdots, p\right\}$. Then $K(0)>0$.

Let $x$ be in $\mathcal{S}_{\gamma}\left(X_{2}\right)$. In the calculation of $L_{\xi_{s} g} g(x)$ we may, and do, replace the polygonal contour of integration $[\xi, \Re(x), x]$ by the polygonal contour $\left[\xi, X_{2}, x\right]$.

Then $L_{\xi_{s}} g(x)=\sum_{j=1}^{j_{s}} b_{s j}\left(I_{s j 1}(x)+I_{s j 2}(x)+I_{s j 3}(x)\right)$, where $b_{s j}=B_{s j}[(j-1) !]^{-1}$, and $I_{s j 1}(x)=\int_{\xi}^{X_{1}} R_{s j}(x, u) d u, I_{s j 2}(x)=\int_{X_{1}}^{X_{2}} R_{s j}(x, u) d u$, and $I_{s j 3}(x)=\int_{X_{2}}^{x} R_{s j}(x, u) d u$, with $R_{s j}(x, u)=g(u) \exp \left[\zeta_{s}(x-u)\right](x-u)^{j-1}$, the contours of integration being straight line segments.

It is easy to see that $I_{8 j 1}(x)=\exp \left[\zeta_{s}\left(x-X_{1}\right)\right] \sum_{t=0}^{j-1} C(j-1, t)\left(x-X_{1}\right)^{t}$ - $\int_{\xi}^{X_{1}} R_{s, j-t}\left(X_{1}, u\right) d u$, where $C(j-1, t)$ is a binomial coefficient. Now $x=X_{1}$ $+\rho e^{i \theta}$, where $\rho>X_{2}-X_{1}$ and where $|\theta|<\gamma$. Hence $\Re\left[\zeta_{s}\left(x-X_{1}\right)\right]=\rho \Re\left(\zeta_{8} e^{i \theta}\right)$ $<-\rho K(\gamma)$. Also $\left|R_{s, j-t}\left(X_{1}, u\right)\right| \leqq B_{0 \xi}[g(x)] e^{-K(0)\left(X_{1}-u\right)}\left(X_{1}-u\right)^{j-t-1}$. Hence

$$
\begin{aligned}
\left|I_{s j 1}(x)\right| & \leqq e^{-\rho K(\gamma)} \sum_{t=0}^{j-1} C(j-1, t) \rho^{t} \int_{\xi}^{X_{1}} B_{0 \xi}[g(x)] e^{-K(0)\left(X_{1}-u\right)}\left(X_{1}-u\right)^{j-t-1} d u \\
& \leqq e^{-\rho K(\gamma)} \sum_{t=0}^{j-1} C(j-1, t) \rho^{t} B_{0 \xi}[g(x)](j-t-1) ![K(0)]^{-j+t}
\end{aligned}
$$

Hence

$$
\left|I_{s j 1}(x)\right| \leqq \delta_{j}\left(X_{2}-X_{1}, \gamma\right) B_{0 \xi}[g(x)],
$$

where $\delta_{j}(X, \gamma)=1$. u.b. $\left\{e^{-\rho K(\gamma)} \sum_{t=0}^{j-1} C(j-1, t) \rho^{t}(j-t-1) ![K(0)]^{-j+t} ; \rho>X\right\}$.

In like manner one can show that

$$
\left|I_{s j 2}(x)\right| \leqq V_{j 1}(\gamma) B_{0 X_{1}}[g(x)] \text {, }
$$

where $V_{j 1}(\gamma)=1 . u . b .\left\{e^{-K(\gamma) r} \sum_{t=0}^{j-1} C(j-1, t) r^{j-1-t}[K(0)]^{-t-1} t ! ; r>0\right\}$, and that 


$$
\left|I_{s ; 3}(x)\right| \leqq\left(B_{\gamma X_{2}}[g(x)]\right) \cdot\left([K(\gamma)]^{-i}(j-1) !\right) .
$$

Relation (25.0) now follows from $(25.1,2,3)$, the quantities $\eta_{s}(X, \gamma), U_{1 s}(\gamma)$, $U_{28}(\gamma)$ being obvious.

26. Lemma 10. Let $\alpha$ be the number defined in $\$ 10$. Let $\gamma$ be any number such that $\alpha \leqq \gamma<\beta$. Let $X$ be any non-negative real number, and let $\phi(x)$ be any function which is analytic in $\mathrm{S}_{\gamma}(X)$ and which has a bounded first derivative in $\mathcal{S}_{\gamma}(X)$. Then $\boldsymbol{K}_{m} \phi(x)$ is bounded in $\mathcal{S}_{\gamma}(X)(m=p+1, p+2, \cdots)$. Moreover, there is a positive number $V_{0}$ independent of $\gamma, X, \phi(x)$, and $m$ such that

$$
B_{\gamma X}\left[K_{m} \phi(x)\right] \leqq m^{-2} V_{0} B_{\gamma X}[D \phi(x)] .
$$

Proof. Let $\rho$ be any non-negative number. Let $T$ be any point on $\mathcal{F}_{m}(\delta)$. Then $|F(T)| \leqq 1 / \lambda(\delta)$, and $|T|^{-1} \leqq(C m)^{-1}$, and $\left|\exp \left[-\rho z_{m} T\right]\right| \leqq e^{-\rho C m}$ $(m=p+1, p+2, \cdots)$, by Lemma 1 . By the same lemma, the length of $\mathcal{F}_{m}(\delta)$ does not exceed $2 \pi(n-1) \delta$. Hence (26.1) holds, with $V_{0}=C^{-2}(n-1) \delta / \lambda(\delta)$.

27. Notation. Using the notations of $\S \S 22$ and 24 , let $g^{*}=F(0)$ $-\sum_{s=1}^{p} P_{s}(0)$.

28. Definition. Using the notations of $\S \S 23$ and 27, let the operator $\boldsymbol{M}_{\xi}$ be defined by the following equation, which is to be valid for every function $\phi(x)$ for which it is meaningful:

$$
\boldsymbol{M}_{\xi} \phi(x)=g^{*} \phi(x)+\sum_{s=1}^{p} L_{\xi_{s}} \phi(x)-\sum_{m=p+1}^{\infty} K_{m} \phi(x) .
$$

29. Lemma 11. Let $\xi$ be any non-negative real number. Let $\phi(x)$ be analytic and bounded, and have a bounded first derivative, in $\oint_{\alpha}(\xi)$, where $\alpha$ is the number defined in $\$ 10$. Then the function $g(x) \equiv M_{\xi} \phi(x)$ is a solution of the difference equation

$$
\sum_{j=1}^{n} A_{j} g\left(x+\omega_{j}\right)=\phi(x) .
$$

Proof. This can be proved directly by the calculus of residues, but in the interest of brevity we shall apply [L: Theorem 2], with $M=0$ and $B=0$, and with $\beta$ replaced by $\alpha$.

Let $\Omega$ be any number greater than $\xi$. Let $g_{1}(x)=g(x+\Omega)$. Let $\phi_{1}(x)$ $=\phi(x+\Omega)$. Then $g_{1}(x)=g_{2}(x)+R(x)$, where $g_{2}(x)=\boldsymbol{M}_{0} \phi_{1}(x)$, and $R(x)$ $=\sum_{s=1}^{p} \int_{\xi-\Omega}^{0}(2 \pi i)^{-1} \int_{J_{s}(\delta)} e^{T(x-u)} \phi_{1}(u) F(T) d T d u$. Now it is easy to see that $R(x)$ is of the form $\sum_{s=1}^{p} \sum_{j=0}^{y_{j}-1} C_{s j} x^{j} \exp \left(\zeta_{s} x\right)$ for some constants $C_{s j}$. Hence $R(x)$ is a solution of the equation $\sum_{j=1}^{n} A_{j} R\left(x+\omega_{j}\right)=0$. Now $\phi_{1}(x)$ is in $\mathcal{C}_{\alpha}(0)$, and is analytic at $x=0$. Hence $\phi_{1}(x)$ is surely of type $(0, \alpha)$, in the notation of [L: p. 439]. Comparison of the function $\boldsymbol{M}_{0} \phi_{1}(x)$, written out in full, with the formulas 41-45 of [L] (cf. also the definition of $\mathcal{L}_{m}$ on page 460 of [L]) shows that in consequence of Theorem 2 of [L] we may conclude that $g_{2}(x)$ is a solution of the equation $\sum_{j=1}^{n} A_{j} g_{2}\left(x+\omega_{j}\right)=\phi_{1}(x)$, whence 
$\sum_{j=1}^{n} A_{j} g_{1}\left(x+\omega_{j}\right)=\phi_{1}(x)$. Hence $\sum_{j=1}^{n} A_{j} g\left(x+\omega_{j}\right)=\phi(x)$.

30. LEMMA 12. There exists a function $W_{1}(\gamma)$ defined for every number $\gamma$ satisfying the conditions $\alpha \leqq \gamma<\beta$, and a number $W_{2}$, such that if $\xi$ is any nonnegative real number, and if $\phi(x)$ is a function which is in $\mathcal{C}_{\gamma}(\xi)$ and has a bounded first derivative in $\mathcal{S}_{\gamma}(\xi)$, then $M_{\xi} \phi(x)$ is in $\mathcal{C}_{\gamma}(\xi)$, and

$$
\boldsymbol{B}_{\gamma \xi}\left[\boldsymbol{M}_{\xi} \phi(x)\right] \leqq W_{1}(\gamma) \boldsymbol{B}_{\gamma \xi}[\phi(x)]+W_{2} \boldsymbol{B}_{\gamma \xi}[\boldsymbol{D} \phi(x)] .
$$

Proof. This follows, with $W_{1}(\gamma)=\left|g^{*}\right|+\sum_{s=1}^{p} V_{s}(\gamma)$, and $W_{2}=\pi^{2} V_{0} / 6$, from Lemmas 9 and 10.

31. Definition. Let the operator $\boldsymbol{A}$ be defined by the following equation, which is to be valid for every function $\phi(x)$ for which it is meaningful:

$$
A \phi(x)=-\left(A_{1}\right)^{-1} \sum_{j=2}^{n} A_{j} \phi\left(x+\omega_{j}\right)\left(^{7}\right) .
$$

32. Notation. Let $r_{0}=\min$ \{distance of $\omega_{j}$ from the boundary of $\mathcal{S}_{\alpha}(0)$; $j=2,3, \cdots, n\}$, where $\alpha$ is the number defined in $\$ 10$.

33. LeMma 13. $r_{0}>0$.

Proof. This follows at once from the definition of $\alpha$.

34. Lemma 14. Let $\gamma$ be any number such that $\alpha \leqq \gamma<\beta$. Let $X$ be any nonnegative real number. Let $\phi(x)$ be in $\mathcal{C}_{\gamma}(X)$. Then $A \phi(x)$ is in $\mathcal{C}_{\gamma}(X)$, and its first derivative is bounded in $\mathrm{S}_{\gamma}(X)$. More precisely, if $A^{*}=\left|A_{1}\right|^{-1} \sum_{j=2}^{n}\left|A_{j}\right|$ and $A^{* *}=A^{*} / r_{0}$, then $B_{\gamma X}[A \phi(x)] \leqq A^{*} B_{\gamma X}[\phi(x)]$ and $B_{\gamma X}[D A \phi(x)]$ $\leqq A^{* *} B_{\gamma X}[\phi(x)]$.

Proof. The first inequality is obvious. The second follows from the fact that as an immediate consequence of the Cauchy integral formula we have

$$
B_{\gamma X}\left[D \phi\left(x+\omega_{j}\right)\right] \leqq B_{\gamma X}[\phi(x)] / r_{0} .
$$

35. Definition. Let the operator $N_{\xi}$ be defined by the following equation, which is to be valid for every function $\phi(x)$ for which it is meaningful:

$$
N_{\xi} \phi(x)=A_{1}^{-1} \phi(x)+M_{\xi} A \phi(x) .
$$

36. LEMMA 15. Let $\gamma$ satisfy the inequalities $\alpha \leqq \gamma<\beta$. Then there is a pasitive number $W(\gamma)$ such that if $\xi$ is any non-negative real number and if $\phi(x)$ is in $\mathcal{C}_{\gamma}(\xi)$, then $N_{\xi} \phi(x)$ is in $\mathcal{C}_{\gamma}(\xi)$, and

$$
\boldsymbol{B}_{\gamma \xi}\left[\boldsymbol{N}_{\xi} \phi(x)\right] \leqq W(\gamma) \boldsymbol{B}_{\gamma \xi}[\phi(x)] .
$$

Proof. This follows at once from Lemmas 12 and 14 ; we may take $W(\gamma)$

(7) It may be worthwhile to emphasize the fact, which is of crucial significance in the sequel, that the summation on the right begins at $j=2$. 
$=\left|A_{1}\right|^{-1}+A^{*} W_{1}(\gamma)+A^{* *} W_{2}$.

37. Lemma 16. Let $\xi$ be a non-negative real number, and let $\phi(x)$ be in $\mathcal{C}_{\alpha}(\xi)$. Then $g(x) \equiv N_{\xi} \phi(x)$ is a solution of the difference equation $\sum_{j=1}^{n} A_{j} g\left(x+\omega_{j}\right)$ $=\phi(x)$.

Proof. This follows from Lemmas 14 and 11.

38. LEMMA 17. Let $\gamma$ satisfy the inequalities $\alpha \leqq \gamma<\beta$. Then there exists a function $\eta(X, \gamma)$, defined for positive $X$ and tending to zero as $X$ becomes infinite, and numbers $U_{1}(\gamma), U_{2}(\gamma)$, such that if $\xi, X_{1}, X_{2}$ are any real numbers for which $0 \leqq \xi<X_{1}<X_{2}$, and if $\phi(x)$ is any function which is in $C_{\alpha}(\xi)$ and in $\mathrm{C}_{\gamma}\left(X_{2}\right)$, then the function $N_{\xi} \phi(x)$ is in $\mathcal{C}_{\gamma}\left(X_{2}\right)$ and

$$
\begin{aligned}
B_{\gamma X_{2}}\left[N_{\xi} \phi(x)\right] \leqq \eta\left(X_{2}\right. & \left.-X_{1}, \gamma\right) B_{\alpha \xi}[\phi(x)] \\
& +U_{1}(\gamma) B_{\alpha X_{1}}[\phi(x)]+U_{2}(\gamma) B_{\gamma X_{2}}[\phi(x)] .
\end{aligned}
$$

Proof. This follows readily, with $\eta(X, \gamma)=A^{*} \sum_{s=1}^{p} \eta_{s}(X, \gamma), U_{1}(\gamma)$ $=A^{*} \sum_{s=1}^{p} \quad U_{1 s}(\gamma), \quad U_{2}(\gamma)=\left|A_{1}\right|^{-1}+A^{*}\left|g^{*}\right|+\pi^{2} A^{* *} V_{0} / 6+A^{*} \sum_{s=1}^{p} U_{2 s}(\gamma)$, from Lemmas 9,10 , and 14 .

39. LеммA 18. If $\phi(x)$ is admissible, then every solution of the difference equation (29.1) which is remotely bounded (cf. §13) is admissible.

Proof. Let $\phi(x)$ be admissible, and let $g(x)$ be a remotely bounded solution of (29.1). Let $\xi$ be a positive number such that $g(x)$ is in $\mathcal{C}_{\alpha}(\xi)$. Let $X$ be any number greater than $\xi$. Now $\sum_{j=1}^{n} A_{j} g(x)=\phi(x)-\sum_{j=1}^{n} A_{j}\left[g\left(x+\omega_{j}\right)-g(x)\right]$. Hence $g(x)=\left(\sum_{j=1}^{n} A_{j}\right)^{-1}\left\{\phi(x)-\sum_{j=1}^{n} A_{j}\left[g\left(x+\omega_{j}\right)-g(x)\right]\right.$. Evidently, as a consequence of the Cauchy integral formula, we have $B_{\alpha X}[D g(x)]$ $\leqq B_{\alpha \xi}[g(x)](\csc \alpha)(X-\xi)^{-1}$, whence $B_{\alpha X}\left[g\left(x+\omega_{j}\right)-g(x)\right] \leqq B_{\alpha \xi}[g(x)](\csc \alpha)$ $\cdot(X-\xi)^{-1}\left|\omega_{j}\right|$. This implies that $g(x)-\left(\sum_{j=1}^{n} A_{j}\right)^{-1} \phi(x)$ approaches zero as $x \rightarrow+\infty$, whence $g(x)$ has a limit as $x \rightarrow+\infty$; thus $g(x)$ is admissible.

40. LемMA 19. If $\phi(x)$ is admissible, and if $\xi$ is any non-negative real number such that $\phi(x)$ is in $\mathcal{C}_{\alpha}(\xi)$, then $N_{\xi} \phi(x)$ is admissible.

Proof. Let $\gamma$ be any number such that $\alpha \leqq \gamma<\beta$. Let $X_{2}$ be larger than $\xi$ and such that $\phi(x)$ is in $\mathcal{C}_{\alpha}\left(X_{2}\right)$. Let $X_{1}$ be chosen arbitrarily so that $\xi<X_{1}$ $<X_{2}$. Then it is evident from (38.1) that $N_{\xi} \phi(x)$ is in $\mathcal{C}_{\gamma}\left(X_{2}\right)$. Thus $N_{\xi} \phi(x)$ is remotely bounded. Hence, by Lemmas 16 and $18, N_{\xi} \phi(x)$ is admissible.

41. ThEOREM 1. If $\phi(x)$ is admissible, then the totality of all admissible solutions of the difference equation

$$
\sum_{\nu=1}^{n} A_{\nu} h\left(x+\dot{v}_{\nu}\right)=\phi(x)
$$

is given by the formula 


$$
h(x)=N_{\xi} \phi(x)+\sum_{s=1}^{p} \sum_{j=0}^{j_{s}-1} C_{s j} x^{j} e^{\zeta_{s} x},
$$

where $\xi$ is any non-negative real number such that $\phi(x)$ is in $\mathcal{C}_{\alpha}(\xi)$, and the $C_{s j}$ are arbitrary constants.

Proof. According to Lemma 8 the function $x^{i} e^{\zeta_{e} x}$ is admissible for every non-negative integer $j$, and it is readily seen that if $j \leqq j_{s}-1$, then $x^{j} e^{\zeta_{s} x}$ is a solution of the homogeneous equation

$$
\sum_{\nu=1}^{n} A_{\nu} g\left(x+\omega_{\nu}\right)=0 .
$$

Hence, by Lemmas 16 and 19, every function of the form (41.2) is an admissible solution of (41.1).

Conversely, if $h(x)$ is any admissible solution of (41.1), then $g(x) \equiv h(x)$ $-\boldsymbol{N}_{\xi} \phi(x)$ is an admissible solution of (41.3); from equation (41.3) itself, solved for $g(x)$ in terms of the $g\left(x+\omega_{\nu}\right)(\nu=2, \cdots, n)$, it is apparent that $g(x)$ can be continued analytically into an integral function $G(x)$, and that $G(x)$ is bounded in every sector $\mathcal{S}_{\gamma}(0)$, with $\gamma<\beta$. Hence $G(x)$ is of type $(0, \beta)$ (in the terminology of [L: p. 439]) and is therefore, by Theorem 2 of [L] (with $\phi(x) \equiv 0$ ), of the form $\sum_{s=1}^{p} \sum_{j=0}^{j_{j}-1} C_{8 j} x^{j} e^{y_{s} x}$.

Part V. The statement of Theorem 2.

\section{Theorem 2. Given the difference equation}

$$
\Lambda\left(x, y\left(x+\omega_{1}\right), \cdots, y\left(x+\omega_{n}\right)\right)=0,
$$

where $\Lambda\left(x, y_{1}, \cdots, y_{n}\right)$ is a polynomial of degree $d \geqq 1$ in the indeterminate $y_{1}, \cdots, y_{n}$, the coefficients being admissible functions of $x$, and where $\omega_{1}=0$ while $\omega_{2}, \cdots, \omega_{n}$ are points of $\delta_{\beta}(0)$. Let $J(\sigma)$ be the polynomial $\Lambda(+\infty, \sigma, \cdots, \sigma)$ in one indeterminate $\sigma$, obtained by replacing every $y_{j}$ $(j=1,2, \cdots, n)$ in $\Lambda\left(+\infty, y_{1}, \cdots, y_{n}\right)$ by the same indeterminate $\sigma$. Let

$$
a_{j}\left(x, y_{1}, \cdots, y_{n}\right)=\partial \Lambda\left(x, y_{1}, \cdots, y_{n}\right) / \partial y_{j}
$$

$(j=1,2, \cdots, n)$. Assume that $J(\sigma)$ is not identically zero, and that its zeros $\left({ }^{8}\right)$ $\sigma_{1}, \sigma_{2}, \cdots, \sigma_{c}$ are all simple zeros. Assume also that $a_{1}\left(+\infty, \sigma_{k}, \cdots, \sigma_{k}\right)$ is different from zero for every $k$ in the set $(1,2, \cdots, c)$. Under these assumptions the totality $\mathcal{T}$ of all admissible solutions of (42.1) can be described as follows:

(a) $\mathcal{T}$ is the union $\mathcal{T}_{1}+\mathcal{T}_{2}+\cdots+\mathcal{T}_{c}$ where $\mathcal{T}_{k}$ is the totality of all admissible solutions $y(x)$ of $(42.1)$ such that $y(+\infty)=\sigma_{k}(k=1,2, \cdots, c)$.

(b) $\mathcal{T}_{k}$ is a nonempty finite-parameter family of functions. More precisely,

( $\left.{ }^{8}\right)$ We do not exclude the case where $J(\sigma)$ reduces to a constant different from zero. In this case the statements below are to be understood as asserting merely that the set $\mathcal{T}$, below, is an empty set. 
if $q_{k}$ is the number $\left(^{9}\right)$ of zeros (multiplicities counted) of the exponential polynomial

$$
f_{k}(z) \equiv \sum_{j=1}^{n} a_{j}\left(+\infty, \sigma_{k}, \sigma_{k}, \cdots, \sigma_{k}\right) e^{\omega_{j} z},
$$

which lie in $\mathcal{N}_{\beta}$, then $\mathcal{T}_{k}$ is a $q_{k}$-parameter family $(k=1,2, \cdots, c)$.

(c) Moreover, $\mathcal{T}$ contains not only every admissible solution of (42.1), but contains also every remotely bounded $\left({ }^{10}\right)$ solution of (42.1).

(By the statement that $\mathcal{T}_{k}$ is a $q_{k}$-parameter family is meant that in terms of a suitable metric (see Part VII below) the space $\mathcal{T}_{k}$ is a manifold of $q_{k}$ complex dimensions, that is, a connected separable metric space each of whose elements has a neighborhood homeomorphic with $q_{k}$-dimensional complex Euclidean space. The number of parameters in $\mathcal{T}_{k}$ is also indicated by the following property of $\mathcal{T}_{k}$ : If $y_{0}(x)$ is any element of $\mathcal{T}_{k}$, then there exist positive numbers $\left.{ }^{11}\right) \xi_{0}, \eta_{0}, E_{0}$, and $\alpha_{0}$, such that $\alpha_{0}<\beta$ and such that if $\xi$ is any number greater than $\xi_{0}$, and if $\lambda_{0}, \lambda_{1}, \cdots, \lambda_{q_{k}-1}$ are any $q_{k}$ complex numbers such that $\left|\lambda_{t}\right|<\eta_{0}\left(t=0,1, \cdots, q_{k}-1\right)$, then there exists one and only one element $y(x)$ of $\mathcal{T}_{k}$ such that $y(x)$ is analytic in $\mathcal{S}_{\alpha_{0}}(\xi)$ and at $x=\xi$, and such that $D^{t} y(\xi)=D^{t} y_{0}(\xi)+\lambda_{t}\left(t=0,1,2, \cdots, q_{k}-1\right)$ and such that $\left|y(x)-y_{0}(x)\right|$ $<E_{0}$ throughout $S_{\alpha_{0}}(\xi)\left({ }^{12}\right)$.)

Proof. The proof is given in Parts VI and VII, below.

43. Generalization. If the hypothesis that $J(\sigma)$ have only simple zeros be dropped, then Parts (a) and (c) of Theorem 2 still hold, and the methods of this paper, exactly as they stand, allow the conclusion to be drawn that for each simple zero $\sigma_{k}$ of $J(\sigma)$, the set $\mathcal{T}_{k}$ satisfies all the statements of Theorem 2, Part (b). However, it is not known to the author whether or not the description of $\tau_{k}$ given in Part b will still apply when $\sigma_{k}$ is a multiple zero.

44. Alternative hypotheses: If each coefficient $C(x)$ in $\Lambda$, instead of being required to be admissible, is required to satisfy the more stringent restriction of being in $\mathcal{C}_{\beta}(\xi)$ for some positive $\xi$ depending upon $C(x)$, and of approaching a limit $C(+\infty)$ in the sense that $B_{\beta X}[C(x)-C(+\infty)]$ tends to zero as $X$ becomes infinite (let us refer to this set of restrictions as Conditions $B$ ), then the set of all solutions of (42.1) which satisfy Conditions B can be given a description closely paralleling that given $\mathcal{T}$ in Theorem 2 , provided however, that in addition to hypotheses analogous to those of Theorem 2 still another hypothesis is assumed, namely that the exponential polynomial $f_{k}(z)$ have no zeros on the boundary of $\mathcal{N}_{\beta}(k=1,2, \cdots, c)$. If this last hypothesis is not assumed, then the set of solutions satisfying Conditions $B$ can fail to answer

\footnotetext{
( ${ }^{9}$ This number is finite, as is seen below.

(10) The definition of remotely bounded is given in $\$ 13$.

(11) The numbers $\eta_{0}, E_{0}$, and $\alpha_{0}$ (but not $\xi_{0}$ ) are independent of $y_{0}(x)$.

(12) When $q_{k}=0, \mathcal{G}_{k}$ contains exactly one function.
} 
such a description. For example, the equation $y(x)-2 y(x+1)=2^{-x}$ has coefficients satisfying Condition $\mathrm{B}$, with $\beta=\pi / 2$, and the $J(\sigma)$ in this case is $-\sigma$, with only one zero (namely, the simple zero $\sigma=0$ ), and $f(z) \equiv 1-2 e^{z}$, with just one zero (namely the negative determination of $\log 1 / 2$ ) in $\mathcal{N}_{\beta}$, which in this example is the nonpositive real axis. There is in this example a one-parameter family of admissible solutions, but no solutions whatever satisfying Conditions B.

\section{Part VI. Existence and uniqueness proofs.}

45. LEMMA 20. If $y(x)$ is any solution of equation (42.1) which is remotely bounded, then $y(x)$ is admissible and $y(+\infty)$ is one of the numbers $\sigma_{k}$ $(k=1,2, \cdots, c)$.

Proof. Let $\epsilon_{j}(x)=y\left(x+\omega_{j}\right)-y(x)(j=1,2, \cdots, n)$. Then $\epsilon_{j}(x)$ tends to zero as $x$ becomes infinite on the positive real axis. (Cf. the discussion in the proof of Lemma 18.) Now $\Lambda\left(x, y\left(x+\omega_{1}\right), \cdots, y\left(x+\omega_{n}\right)\right)=\Lambda(x, y(x)$ $\left.+\epsilon_{1}(x), \cdots, y(x)+\epsilon_{n}(x)\right)=\Lambda(x, y(x), y(x), \cdots, y(x))+\sum_{0} \epsilon_{1}^{s_{1}}(x) \cdots \epsilon_{n}^{s_{n}}(x)$ $C\left[s_{1}, \cdots, s_{n} ; x ; y(x), y(x), \cdots, y(x)\right]=0$, where $\sum_{0}$ is the summation over all indices $s_{1}, \cdots, s_{n}$ such that $1 \leqq s_{1}+\cdots+s_{n} \leqq d$ and

$$
\begin{gathered}
C\left[s_{1}, \cdots, s_{n} ; x ; y_{1}, \cdots, y_{n}\right] \\
=\frac{\partial^{s_{1}+\cdots+s_{n}} \Lambda\left(x, y_{1}, \cdots, y_{n}\right) / \partial y_{1}^{s_{1}} \cdots \partial y_{n}^{s_{n}}}{s_{1} ! \cdots s_{n} !} .
\end{gathered}
$$

Letting $P(x, y)$ be the polynomial in $y$ defined as $\Lambda(x, y, y, \cdots, y)$ $+\sum_{0} \epsilon_{1}^{s_{1}}(x) \cdots \epsilon_{n}^{s_{n}}(x) C\left[s_{1}, \cdots, s_{n} ; x ; y, y, \cdots, y\right]$, we see that the coefficients in $P(x, y)$ are, when $x$ is large and positive, arbitrarily near the coefficients in $\Lambda(+\infty, y, \cdots, y)$ so that when $x$ is large and positive, $y(x)$ is either near the set $\left(\sigma_{1}, \sigma_{2}, \cdots, \sigma_{c}\right)$ or else is very large (a possibility, at first glance, if the initial coefficient of $\Lambda(x, y, \cdots, y)$ tends to zero as $x$ becomes positively infinite). Since $y(x)$ is remotely bounded, the possibility that $y(x)$ be very large is excluded. Since $y(x)$ is continuous, it must, for all sufficiently large $x$, be near a fixed one of the numbers $\sigma_{k}$.

46. Lemma 21. Parts (a) and (c) of Theorem 2 are valid.

Proof. This is an immediate consequence of Lemma 20.

47. Lemma 22. Let $k$ be any one of the numbers $1,2, \cdots, c$. Then equation (42.1) is equivalent, under the transformation $y(x)=\sigma_{k}+h(x)$, to the equation

$$
\begin{aligned}
\sum_{j=1}^{n} a_{k j} h\left(x+\omega_{j}\right)= & \psi_{k}(x)+\sum_{j=1}^{n} e_{k j}(x) h\left(x+\omega_{j}\right) \\
& +\sum_{1} g_{k}\left(i_{1}, \cdots, i_{n} ; x\right) h^{i_{1}}\left(x+\omega_{1}\right) \cdots h^{i_{n}}\left(x+\omega_{n}\right)
\end{aligned}
$$

where 


$$
\begin{aligned}
& a_{k j}=a_{j}\left(+\infty, \sigma_{k}, \cdots, \sigma_{k}\right) \\
& \psi_{k}(x)=-\Lambda\left(x, \sigma_{k}, \cdots, \sigma_{k}\right), \\
& e_{k j}(x)=a_{j}\left(+\infty, \sigma_{k}, \cdots, \sigma_{k}\right)-a_{j}\left(x, \sigma_{k}, \cdots, \sigma_{k}\right), \\
& g_{k}\left(i_{1}, \cdots, i_{n} ; x\right)=-C\left[i_{1}, \cdots, i_{n} ; x ; \sigma_{k}, \sigma_{k}, \cdots, \sigma_{k}\right]
\end{aligned}
$$

(cf. (45.1)), and

(47.6) $\sum_{1}$ is the summation over all indices $i_{1}, \cdots, i_{n}$ such that $2 \leqq i_{1}$ $+\cdots+i_{n} \leqq d$.

Proof. This is obvious.

48. LEMMA 23. The functions $\psi_{k}(x), e_{k j}(x)$, and $g_{k}\left(i_{1}, \cdots, i_{n} ; x\right)$ are admissible, and $\psi_{k}(+\infty)=e_{k j}(+\infty)=0(j=1,2, \cdots, n ; k=1,2, \cdots, c)$. Also $\sum_{j=1}^{n} a_{k j} e^{\omega_{j z}} \equiv f_{k}(z)$, where $f_{k}(z)$ is defined by (42.3).

Proof. This is obvious.

49. Definition. Let $k$ be in the set $(1,2, \cdots, c)$. Let $Q_{k}\left(x, h_{1}, \cdots, h_{n}\right)$ be the polynomial in indeterminates $h_{1}, \cdots, h_{n}$, with coefficients functions of $x$, defined by the equation

$$
\begin{aligned}
Q_{k}\left(x, h_{1}, h_{2}, \cdots, h_{n}\right) \equiv \psi_{k}(x) & +\sum_{j=1}^{n} e_{k j}(x) h_{j} \\
& +\sum_{1} g_{k}\left(i_{1}, \cdots, i_{n} ; x\right) h_{1}^{i_{1}} \cdots h_{n}^{i_{n}} .
\end{aligned}
$$

50. Definition. Let the nonlinear difference operator $\boldsymbol{Q}_{k}$ be defined by the following equation, which is to be valid for every function $h(x)$ for which it is meaningful:

$$
Q_{k} h(x)=Q_{k}\left(x, h\left(x+\omega_{1}\right), \cdots, h\left(x+\omega_{n}\right)\right) .
$$

51. Definition. In the sequel we shall use a fixed value of $k$ in the set $(1,2, \cdots, c)$, and define $A_{j}=a_{k j}(j=1,2, \cdots, n)$. The operators $N_{\xi}$ we then define in terms of the $A_{j}$ and $\omega_{j}$ as in Part IV. Certain of the functions to be used in the sequel will be designated by symbols which do not display the fact that the function depends upon the choice of $k$.

52. Lemma 24. Let $y(x)$ be an admissible solution of equation (42.1). Let $y(x)=\sigma_{k}+h(x)$. If $\xi$ is any non-negative real number sufficiently large so that $y(x)$ is in $\mathcal{C}_{\alpha}(\xi)$ and so that the coefficients in $\Lambda$ are in $\mathcal{C}_{\alpha}(\xi)$, then

$$
h(x)=N_{\xi} Q_{k} h(x)+\sum_{s=1}^{p} \sum_{j=0}^{j_{8}-1} C_{s j} x^{j} e^{\zeta_{s} x}
$$

for some constants $C_{s j}$.

Proof. This follows at once from Theorem 1 and equation (47.1). It may be noted that the hypotheses $\left[A_{1} \neq 0\right],\left[\sum_{j=1}^{n} A_{j} \neq 0\right]$ (cf. Lemma 1) of Theorem 
1 are equivalent, respectively, to the hypotheses $\left[a_{1}\left(+\infty, \sigma_{k}, \cdots, \sigma_{k}\right) \neq 0\right]$, $\left[\sigma_{k}\right.$ is simple] of Theorem 2 .

53. Notation. Let $\xi_{1}$ be the greatest lower bound of all non-negative real numbers $X$ such that every coefficient in $Q_{k}\left(x, h_{1}, \cdots, h_{n}\right)$ is in $C_{\alpha}(X)$, and such that

$$
B_{\alpha X}\left[e_{k j}(x)\right] \leqq[2 n W(\alpha)]^{-1} \quad(j=1,2, \cdots, n),
$$

where $W(\alpha)$ is defined in $\S \S 10$ and 36. Let $\xi_{2}=1+\xi_{1}$.

54. Definition. Let $\xi$ be any real number such that $\xi \geqq \xi_{2}$. Let $\mathfrak{F}=\left\{C_{s j m} ; s=1,2, \cdots, p ; j=0,1, \cdots, j_{s}-1 ; m=0,1,2, \cdots\right\}$ be a sequence of complex numbers. Then by the sequence $\left\{h_{m}(x, \xi, \mathfrak{F})\right.$; $m=0,1, \cdots\}$ will be meant the sequence of functions defined recursively by the equations

$$
\begin{aligned}
h_{0}(x, \xi, \mathfrak{F})= & \sum_{s=1}^{p} \sum_{j=0}^{j_{s}-1} C_{s j 0}(x-\xi)^{j} e^{\xi_{s}(x-\xi)}, \\
h_{m+1}(x, \xi, \mathfrak{F})= & N_{\xi} Q_{k} h_{m}(x, \xi, \mathfrak{F}) \\
& \quad+\sum_{s=1}^{p} \sum_{j=0}^{j_{r}-1} C_{s, j, m+1}(x-\xi)^{j e_{s}(x-\xi)} .
\end{aligned}
$$

55. Notation. If $\mathbb{S}$ is any bounded sequence of complex numbers (finite or infinite), then by $U[\mathcal{S}]$ is meant the least upper bound of the moduli of the terms of $(B)$.

56. Notation. For any $\xi$ which is greater than or equal to $\xi_{2}$ the symbol $\delta_{\xi}$ represents $B_{\alpha \xi}\left[\psi_{k}(x)\right]$.

57. LEMMA 25. Let $M$ be any non-negative real number, let $\theta$ be any positive number less than unity, and let $d$ be any positive integer. Then for every positive $\epsilon$ there is a largest positive $\delta$ such that the equation

$$
X=\delta+\theta X+M\left(X^{2}+X^{3}+\cdots+X^{d}\right)
$$

has a positive solution less than or equal to $\epsilon$.

Proof. This is obvious.

58. LeMmA 26. Let $\sum_{m=0}^{\infty} \epsilon_{m}$ be a convergent sequence of non-negative real numbers and let $r$ be a positive number less than unity. Let $\left\{a_{m}\right\}$ be a sequence of non-negative numbers satisfying the inequalities

$$
a_{m+1} \leqq r a_{m}+\epsilon_{m} \quad(m=0,1,2, \cdots) .
$$

Then $\sum_{m=0}^{\infty} a_{m}$ converges.

Proof. Let $s_{m}=a_{0}+a_{1}+\cdots+a_{m}$. Then $s_{m+1} \leqq r s_{m}+\left(\epsilon_{0}+\cdots+\epsilon_{m}\right)$ $+a_{0}$. Let $M_{0}=a_{0}+\sum_{m=0}^{\infty} \epsilon_{m}$. Then $s_{m+1} \leqq r s_{m}+M_{0}$. Hence $s_{m+1} \leqq s_{0} r^{m+1}$ 
$+M_{0}\left(1+r+\cdots+r^{m}\right) \leqq s_{0}+M_{0} /(1-r)$. Hence $\sum_{m=0}^{\infty} a_{m}$ converges.

59. Lemma 27. For every positive number $E$ there exists $\left({ }^{13}\right)$ a positive number $\rho_{E}$ and a positive number $\eta_{E}$ such that if $\xi \geqq \xi_{2}$, and if $\delta_{\xi} \leqq \rho_{E}$, and if $\mathfrak{F}$ is any bounded sequence of complex numbers such that $U[\mathfrak{F}] \leqq \eta_{E}$, then the sequence $\left\{h_{m}(x, \xi, \mathfrak{F})\right\}$ satisfies the inequalities

$$
B_{\alpha \xi}\left[h_{m}(x, \xi, \mathfrak{F})\right] \leqq E \quad(m=1,2, \cdots) .
$$

Proof. Let $r_{m}(x, \xi, \mathfrak{F})=\sum_{s=1}^{p} \sum_{j=0}^{j_{s}-1} C_{s j m}(x-\xi)^{j} e^{\xi_{s}(x-\xi)}$, let $H_{m}=B_{\alpha \xi}\left[h_{m}(x, \xi\right.$, $\mathfrak{F})$, and let $R_{m}=B_{\alpha \xi}\left[r_{m}(x, \xi, \mathfrak{F})\right](m=0,1,2, \cdots)$. Let

$$
\delta_{2}=\max \left\{B_{\alpha \xi_{2}}\left[e_{k j}(x)\right] ; j=1,2, \cdots, n\right\},
$$

let

$$
M_{2}=\max \left\{B_{\alpha \xi_{2}}\left[g_{k}\left(i_{1}, \cdots, i_{n} ; x\right)\right] ; 2 \leqq i_{1}+\cdots+i_{n} \leqq d\right\}
$$

let

$$
b=\max \left\{\text { number of terms of degree } s \text { in } \sum_{1} ; s=2,3, \cdots, d\right\},
$$

and let

$$
G(\alpha)=\sum_{s=1}^{p} \sum_{j=0}^{j_{s}-1} G_{s j}(\alpha),
$$

where the $G_{s j}(\alpha)$ are defined in Lemma 8. Then

$$
H_{0}=R_{0} \leqq G(\alpha) U[\mathfrak{F}] \text {. }
$$

Also

$$
H_{m+1} \leqq B_{\alpha \xi}\left[N_{\xi} Q_{k} h_{m}(x, \xi, \mathfrak{F})\right]+R_{m+1}
$$

Hence, by Lemma 15, we have

$$
H_{m+1} \leqq W(\alpha) B_{\alpha \xi}\left[Q_{k} h_{m}(x, \xi, \mathfrak{F})\right]+R_{m+1}
$$

Therefore

$$
H_{m+1} \leqq W(\alpha)\left\{\delta_{\xi}+n \delta_{2} H_{m}+M_{2} b\left(H_{m}^{2}+\cdots+H_{m}^{d}\right)\right\}+G(\alpha) U[\mathfrak{F}] .
$$

Now let $E$ be any positive number. Let $\theta=1 / 2$. Let $\delta$ be the largest positive number such that the equation $t=\delta+\theta t+M_{2} b W(\alpha) \cdot\left(t^{2}+t^{3}+\cdots+t^{d}\right)$ has a positive solution $t_{0}$ less than or equal to $E$. (Cf. Lemma 25, with $M=M_{2} b W(\alpha)$.) Obviously $t_{0}>\delta$.

${ }^{\left({ }^{13}\right)}$ In this lemma $\rho_{E}, \eta_{E}$ are asserted to exist. Plainly they are not unique. However, in the application of this lemma, where we wish to make use of a particular choice of $\rho_{E}$ and $\eta_{E}$, we shall refer, loosely, to the $\rho_{E}$ and $\eta_{E}$ of Lemma 27 , meaning thereby that a particular choice from among the infinitely many possibilities is made, once for all. This remark applies to several other lemmas following this. 
Let $\rho_{E}=\delta /(2 W(\alpha))$. Let $\eta_{E}=\delta(2 G(\alpha))\left({ }^{14}\right)$. Then if $\xi \geqq \xi_{2}$, and if $\delta_{\xi} \leqq \rho_{E}$, and if $U[\mathfrak{F}] \leqq \eta_{E}$, we have

$$
H_{0} \leqq \delta / 2<t_{0}
$$

and also, using (53.1), we have

$$
H_{m+1} \leqq \delta+\theta H_{m}+M_{2} b W(\alpha)\left(H_{m}^{2}+\cdots+H_{m}^{d}\right),
$$

so that if $H_{m} \leqq t_{0}$, then $H_{m+1} \leqq \delta+\theta t_{0}+M_{2} b W(\alpha)\left(t_{0}^{2}+\cdots+t_{0}^{d}\right)=t_{0}$. Thus by complete induction we have $H_{m} \leqq t_{0}<E(m=0,1,2, \cdots)$.

60. LeMMA 28. Let $E$ and $\epsilon$ be positive numbers, with $\epsilon \leqq E$. There exists a positive number $W_{0}=W_{0}(E, \epsilon)$ such that if $\rho_{E}$ and $\eta_{E}$ are the numbers of Lemma 27 , and if $\xi$ is any number greater than or equal to $\xi_{2}$, for which $\delta_{\xi} \leqq \rho_{E}$, and if $\boldsymbol{U}[\mathfrak{F}] \leqq \eta_{E}$, then $\boldsymbol{B}_{\alpha X}\left[h_{m}(x, \xi, \mathfrak{F})\right] \leqq \epsilon$ whenever $X \geqq \xi+W_{0}(m=0,1, \cdots)$.

Proof. Let $\xi$ be any number such that $\xi \geqq \xi_{2}$ and $\delta_{\xi} \leqq \rho_{E}$. Let $U[\mathfrak{F}] \leqq \eta_{E}$. From Lemma 16 , and the fact that $r_{m+1}(x, \xi, \mathfrak{F})$ is evidently a solution of the difference equation $\sum_{j=1}^{n} A_{j} g\left(x+\omega_{j}\right)=0$, we have that $\sum_{j=1}^{n} A_{j} h_{m+1}\left(x+\omega_{j}, \xi, \mathfrak{F}\right)$ $=Q_{k} h_{m}(x, \xi, \mathfrak{F})$. Hence

$$
\begin{aligned}
\sum_{j=1}^{n} A_{j} h_{m+1}(x, \xi, \mathfrak{F})=Q_{k} h_{m}(x, \xi, \mathfrak{F}) & \\
& +\sum_{j=1}^{n} A_{i}\left[h_{m+1}(x, \xi, \mathfrak{F})-h_{m+1}\left(x+\omega_{j}, \xi, \mathfrak{F}\right)\right] .
\end{aligned}
$$

Let $X$ be any number such that $X>\xi$. Then, by the Cauchy integral formula we have $\boldsymbol{B}_{\alpha X}\left[D h_{m+1}(x, \xi, \mathfrak{F})\right] \leqq(X-\xi)^{-1} \csc \alpha \cdot \boldsymbol{B}_{\alpha \xi}\left[h_{m+1}(x, \xi, \mathfrak{F})\right]$, whence $\boldsymbol{B}_{\alpha X}\left[h_{m+1}(x, \xi, \mathfrak{F})-h_{m+1}\left(x+\omega_{j}, \xi, \mathfrak{F}\right)\right] \leqq(X-\xi)^{-1}(\csc \alpha) E\left|\omega_{j}\right|$. Hence, letting $a^{*}=\left|\sum_{j=1}^{n} A_{j}\right|^{-1}$, we have, from (60.1),

$$
B_{\alpha X}\left[h_{m+1}(x, \xi, \mathfrak{F})\right] \leqq a^{*}\left\{B_{\alpha X}\left[Q_{k} h_{m}(x, \xi, \mathfrak{F})\right]\right.
$$

$$
\left.+E(X-\xi)^{-1} \csc \alpha \sum_{j=1}^{n}\left|A_{j}\right|\left|\omega_{i}\right|\right\} \text {. }
$$

Thus, letting $M_{2}$ be defined by (59.3), letting $R=R(W)=\sum_{j=1}^{n}\left|A_{j}\right|\left|\omega_{j}\right|$ $\cdot(E \csc \alpha) W^{-1}$, letting $S=S(X)=\max \left\{B_{\alpha X}\left[\psi_{k}(x)\right], \quad B_{\alpha X}\left[e_{k 1}(x)\right], \cdots\right.$, $\left.B_{\alpha X}\left[e_{k n}(x)\right]\right\}$, letting $T=T(W)=\max B_{\alpha W}\left[x^{j} e^{\zeta_{s} x}\right] ; j=0,1, \cdots, j_{s}-1$; $s=1,2, \cdots, p\}$, and letting $v_{m}=B_{\alpha X}\left[h_{m+1}(x, \xi, \mathfrak{F})\right]$, we have

$$
v_{0} \leqq B_{\alpha X}\left[r_{0}(x, \xi, \mathfrak{F})\right] \leqq \eta_{E} T(X-\xi) \text {, }
$$

and from (60.2) we have

(60.4) $v_{m+1} \leqq a^{*}[S(X)+R(X-\xi)]+a^{*} n S(X) v_{m}+a^{*} M_{2} b\left(v_{m}^{2}+\cdots+v_{m}^{d}\right)$.

(14) If $p=0$, there is no sequence $\mathfrak{F}$ and no number $G(\alpha)$. The changes of language needed to cover this case are, however, sufficiently obvious. 
We note that $S(X)$ tends to zero as $X$ becomes infinite. (Cf. Lemmas 23 and 4.) We note also that $R(W)$ and $T(W)$ tend to zero as $W$ becomes infinite. (Cf. Lemma 8.)

Let $\theta=1 / 2$. Let $\delta$ be the smallest positive number such that the equation

$$
t=\delta+\theta t+a^{*} M_{2} b\left(t^{2}+\cdots+t^{d}\right)
$$

has a positive solution $t_{0}$ less than or equal to $\epsilon$. Let $W_{3}$ be the smallest positive number such that $W_{3} \geqq 1$, and such that $\eta_{E} T\left(W_{3}\right) \leqq t_{0}$, and such that $a^{*} R\left(W_{3}\right)$ $\leqq \delta_{2}$. Let $X_{3}$ be the smallest positive number such that $a^{*} S\left(X_{3}\right) \leqq \delta / 2$ and $a^{*} n S\left(X_{3}\right) \leqq \theta$. Let $W_{0}=\max \left[W_{3}, X_{3}-\xi_{2}\right]$. Then if $X \geqq \xi+W_{0}$, we have $X \geqq X_{3}$, so that $a^{*} S(X) \leqq \delta / 2$ and $a^{*} n S(X) \leqq \theta$. Also, if $X \geqq \xi+W_{0}$, then $X-\xi \geqq W_{3}$, so that $a^{*} R(X-\xi) \leqq \delta / 2$ and, using (60.3), $v_{0} \leqq \eta_{E} T\left(W_{3}\right)$. Thus $v_{0} \leqq t_{0}$, and if $v_{m} \leqq t_{0}$, then, by (60.4), we have $v_{m+1} \leqq \delta+\theta t_{0}+a^{*} M_{2} b\left(t_{0}^{2}+\cdots\right.$ $\left.+t_{0}^{d}\right)=t_{0}$, so that $v_{m} \leqq \epsilon(m=0,1, \cdots)$.

Thus this number $W_{0}$ has the required properties.

61. LemMA 29. $Q_{k}\left(x, h_{1}, \cdots, h_{n}\right)-Q_{k}\left(x, l_{1}, \cdots, l_{n}\right)=\sum_{j=1}^{n} e_{k j}(x)\left(h_{j}-l_{j}\right)$ $+\sum_{2} G_{k j}\left(s_{1}, \cdots, s_{n} ; t_{1}, \cdots, t_{n} ; x\right)\left(h_{j}-l_{j}\right)\left(h_{1}^{s_{1}} h_{2}^{s_{2}} \ldots h_{n}^{s_{n}}\right)\left(l_{1}^{t_{1}} l_{2}^{t_{2}} \cdots l_{n}^{l_{n}}\right)$, where $\sum_{2}$ is the summation over a certain set of indices $j ; s_{1}, \cdots, s_{n}$; $t_{1}, \cdots, t_{n}$ such that $j$ is in the set $(1,2, \cdots, n)$ and such that $1 \leqq s_{1}+\cdots$ $+s_{n}+t_{1}+\cdots+t_{n} \leqq d-1$, and where $G_{k j}\left(s_{1}, \cdots, s_{n} ; t_{1}, \cdots, t_{n} ; x\right)$ is one of the functions $g_{k}\left(i_{1}, \cdots, i_{n} ; x\right)$.

Proof. This is obvious.

62. Notation. Let $b_{2}$ be the total number of terms in $\sum_{2}$.

63. Notation. Let $E_{0}$ be the largest positive number such that $b_{2} M_{2}\left(E_{0}+E_{0}^{2}+\cdots+E_{0}^{d-1}\right) \leqq 1 / 4$.

64. Lemma 30. Let $E$ be any positive number less than or equal to $E_{0}$. Let $\rho_{E}$ and $\eta_{E}$ be the numbers of Lemma 27. Let $\xi$ be any number greater than or equal to $\xi_{2}$ and such that $\delta_{\xi} \leqq \rho_{E}$, let $\mathfrak{F}$ satisfy the condition $U[\mathfrak{F}] \leqq \eta_{E}$, and let it be given that $\mathfrak{F}$ satisfies the additional condition that for each fixed pair $s, j$ the series $\sum_{m=0}^{\infty}\left|C_{s j, m+1}-C_{s j m}\right|$ converges. Then the sequence $\left\{h_{m}(x, \xi, \mathfrak{F})\right\}$ converges uniformly in $\mathrm{S}_{\alpha}(\xi)$.

Proof. Let $\Delta h_{m}(x, \xi, \mathfrak{F})=h_{m+1}(x, \xi, \mathfrak{F})-h_{m}(x, \xi, \mathfrak{F}), \Delta r_{m}(x, \xi, \mathfrak{F})$ $=r_{m+1}(x, \xi, \mathfrak{F})-r_{m}(x, \xi, \mathfrak{F}), Y_{m}=B_{\alpha \xi}\left[\Delta h_{m}(x, \xi, \mathfrak{F})\right]$, and $\epsilon_{m}=B_{\alpha \xi}\left[\Delta r_{m}(x, \xi, \mathfrak{F})\right]$ $(m=0,1, \cdots)$. For brevity, we shall omit the arguments $\xi$, $\mathfrak{F}$ in the following equations.

Now $\Delta h_{m}(x)=\boldsymbol{N}_{\xi}\left(\boldsymbol{Q}_{k} h_{m}(x)-\boldsymbol{Q}_{k} h_{m-1}(x)\right)+\Delta r_{m}(x)$. Thus, by Lemma 29 we have $\Delta h_{m}(x)=N_{\xi}\left\{\sum_{j=1}^{n} e_{k j}(x) \Delta h_{m-1}\left(x+\omega_{j}\right)+\sum_{2} G_{k j}\left(s_{1}, \cdots, s_{n} ; t_{1}, \cdots\right.\right.$, $\left.\left.t_{n} ; x\right) \Delta h_{m-1}\left(x+\omega_{j}\right) h_{m}^{s_{1}}\left(x+\omega_{1}\right) \cdots h_{m}^{s_{n}}\left(x+\omega_{n}\right) h_{m-1}^{t_{1}}\left(x+\omega_{1}\right) \cdots h_{m-1}^{t_{n}}\left(x+\omega_{n}\right)\right\}$ $+\Delta r_{m}(x)$. Therefore, using the notations of Lemmas 15 and 27, we have

$$
Y_{m} \leqq W(\alpha)\left\{n \delta_{2} Y_{m-1}+\sum_{2} M_{2} Y_{m-1} E^{s_{1}}+\cdots+s_{n}+t_{1}+\cdots+t_{n}\right\}+\epsilon_{m},
$$


and therefore we have

$$
Y_{m} \leqq Y_{m-1}\left\{n \delta_{2} W(\alpha)+b_{2} M_{2}\left(E_{0}+E_{0}^{2}+\cdots+E_{0}^{d-1}\right)\right\}+\epsilon_{m} .
$$

Now $n \delta_{2} W(\alpha) \leqq 1 / 2$, by $(53.1)$, and $b_{2} M_{2}\left(E_{0}+\cdots+E_{0}^{d-1}\right) \leqq 1 / 4$, by $\S 63$. Hence $n \delta_{2} W(\alpha)+b_{2} M_{2}\left(E_{0}+\cdots+E_{0}^{d-1}\right) \leqq 3 / 4$. Hence, by Lemma 26 , $\sum_{m=0}^{\infty} Y_{m}$ converges. This implies that the sequence $\left\{h_{m}(x, \xi, \mathfrak{F})\right\}$ converges uniformly in $\mathcal{S}_{\alpha}(\xi)$.

65. Lemma 31. Let $E_{0}$ be the positive number defined in $\$ 63$. Let $E$ be a positive number less than or equal to $E_{0}$. Let $\rho_{E}, \eta_{E}$ be the numbers of Lemma 27. Let $\xi$ be a number such that $\xi \geqq \xi_{2}$ and $\delta_{\xi} \leqq \rho_{E}$. Let $\mathfrak{F}$ be a sequence $\left\{C_{s j m}\right\}$ such that $U[\mathfrak{F}] \leqq \eta_{E}$, and such that for each $s, j$ the series $\sum_{m=0}^{\infty}\left|C_{s j, m+1}-C_{s j m}\right|$ converges.

Then for every positive $\epsilon$ and for every $\gamma$ greater than $\alpha$ and less than $\beta$ there is a positive $X^{\prime \prime}=X^{\prime \prime}(\gamma, \epsilon)$, such that $h_{m}(x, \xi, \mathfrak{F})$ is analytic throughout $\mathcal{S}_{\gamma}\left(X^{\prime \prime}\right)$ and $B_{\gamma X^{\prime \prime}}\left[h_{m}(x, \xi, \mathfrak{F})\right]<\epsilon$.

Proof. The asserted analyticity of $h_{m}(x, \xi, \mathfrak{F})$ follows readily from the nature of the operators $\boldsymbol{Q}_{k}$ and $\boldsymbol{N}_{\xi}$. We shall consider, therefore only the estimation of $\boldsymbol{B}_{\alpha X^{\prime \prime}}\left[h_{m}(x, \xi, \mathfrak{F})\right]$.

Let $X^{*} \geqq \xi_{2}$ be such that every $g_{k}\left(i_{1}, \cdots, i_{n} ; x\right)$ is in $\mathcal{C}_{\gamma}\left(X^{*}\right)$. Let $M^{\prime \prime}$ $=\max \left\{B_{\gamma X^{*}}\left[g_{k}\left(i_{1}, \cdots, i_{n} ; x\right)\right] ; 2 \leqq i_{1}+i_{2}+\cdots+i_{n} \leqq d\right\}$. Let $X^{\prime}$ and $X^{\prime \prime}$ be chosen as any numbers such that $\xi<X^{\prime}<X^{\prime \prime}$, and such that $X^{\prime}>X^{*}$; these two numbers will be subjected to further restrictions in the sequel.

Then, using Lemma 17, we have

$$
\begin{aligned}
\boldsymbol{B}_{\gamma X^{\prime \prime}}\left[h_{m+1}(x, \xi, \mathfrak{F})\right] \leqq & \eta\left(X^{\prime \prime}-X^{\prime}, \gamma\right) \boldsymbol{B}_{\alpha \xi}\left[\boldsymbol{Q}_{k} h_{m}(x, \xi, \mathfrak{F})\right] \\
& +U_{1}(\gamma) B_{\alpha X^{\prime}}\left[\boldsymbol{Q}_{k} h_{m}(x, \xi, \mathfrak{F})\right] \\
& +U_{2}(\gamma) B_{\gamma X^{\prime \prime}}\left[\boldsymbol{Q}_{k} h_{m}(x, \xi, \mathfrak{F})\right] \\
& +B_{\gamma X^{\prime \prime}}\left[r_{m+1}(x, \xi, \mathfrak{F})\right] .
\end{aligned}
$$

Letting $\delta_{2}, M_{2}, b$ be defined, as usual, by (59.2), (59.3), and (59.4), and letting $J_{m}=B_{\gamma X^{\prime \prime}}\left[h_{m}(x, \xi, \mathfrak{F})\right], L=$ l.u.b. $\left\{B_{\alpha X^{\prime}}\left[h_{m}(x, \xi, \mathfrak{F})\right] ; m\right.$ $=0,1,2, \cdots\}, \delta^{*}=\max \left\{B_{\alpha X^{\prime}}\left[\psi_{k}(x)\right], B_{\alpha X^{\prime}}\left[e_{k 1}(x)\right], \cdots, B_{\alpha X^{\prime}}\left[e_{k n}(x)\right]\right\}$, $\delta^{* *}=\max \left\{\boldsymbol{B}_{\gamma X^{\prime \prime}}\left[\psi_{k}(x)\right], \boldsymbol{B}_{\gamma X^{\prime \prime}}\left[e_{k 1}(x)\right], \cdots, \boldsymbol{B}_{\gamma X^{\prime \prime}}\left[e_{k n}(x)\right]\right\}, \rho=$ l.u.b. $\left\{\boldsymbol{B}_{\gamma X^{\prime \prime}}\right.$ $\left.\cdot\left[r_{m+1}(x, \xi, \mathfrak{F})\right], m=0,1, \cdots\right\}$, we have $J_{0} \leqq \rho$ and

$$
J_{m+1} \leqq C_{0}+C_{1} J_{m}+C_{2}\left(J_{m}^{2}+J_{m}^{3}+\cdots+J_{m}^{d}\right)
$$

where

$$
\begin{aligned}
C_{0}= & \eta\left(X^{\prime \prime}-X^{\prime}, \gamma\right)\left\{\delta_{\xi}+n \delta_{2} E_{0}+M_{2} b\left(E_{0}^{2}+E_{0}^{3}+\cdots+E_{0}^{d}\right)\right. \\
& +U_{1}(\gamma)\left\{\delta^{*}+n \delta^{*} L+M_{2} b\left(L^{2}+L^{3}+\cdots+L^{d}\right)\right\} \\
& +U_{2}(\gamma) \delta^{* *}+\rho,
\end{aligned}
$$


where $C_{1}=U_{2}(\gamma) n \delta^{* *}$, and where $C_{2}=U_{2}(\gamma) M^{\prime \prime} b$.

Let $\epsilon$ be any positive number. Let $\theta=1 / 2$. Let $\delta$ be a positive number such that the equation $t=\delta+\theta t+C_{2}\left(t^{2}+t^{3}+\cdots+t^{d}\right)$ has a positive solution $t_{0}$ less than $\epsilon$. Obviously $t_{0}>\delta$. Let $X^{\prime}$ be so large that $U_{1}(\gamma)\left\{\delta^{*}+n \delta^{*} L\right.$ $\left.+M_{2} b\left(L^{2}+L^{3}+\cdots+L^{d}\right)\right\}<\delta / 2$. (Cf. Lemma 28.) Then let $X^{\prime \prime}$ be so large that $\eta\left(X^{\prime \prime}-X^{\prime}, \gamma\right) \cdot\left\{\delta_{\xi}+n \delta_{2} E_{0}+M_{2} b\left(E_{0}^{2}+E_{0}^{3}+\cdots+E_{0}^{d}\right)\right\}<\delta / 6$, and $U_{2}(\gamma) \delta^{* *}<\delta / 6$, and $\rho<\delta / 6$, and $U_{2}(\gamma) n \delta^{* *}<\theta$. Then $J_{0} \leqq \delta / 6<t_{0}$, and $J_{m+1} \leqq \delta+\theta J_{m}+C_{2}\left(J_{m}^{2}+J_{m}^{3}+\cdots+J_{m}^{d}\right)$, so that by complete induction we have $J_{m} \leqq t_{0}(m=0,1,2, \cdots)$. Hence $J_{m}<\epsilon$. That is, $B_{\gamma X^{\prime \prime}}\left[h_{m}(x, \xi, \mathfrak{F})\right]<\epsilon$ $(m=0,1, \cdots)$.

66. Lemma 32. Let $E_{0}, E, \rho_{E}, \eta_{E}, \xi$, and $\mathfrak{F}$ satisfy the hypotheses of Lemma 31. Then the sequence $\left\{h_{m}(x, \xi, \mathfrak{F})\right\}$ converges, with local uniformity, in a region $R$ which, for every positive $\gamma$ less than $\beta$, contains a sector $\mathcal{S}_{\gamma}(X(\gamma))$, for some nonnegative number $X(\gamma)$. The limit function $h(x, \xi, \mathfrak{F})$ of the sequence $\left\{h_{m}(x, \xi, \mathfrak{F})\right\}$ is admissible and is a solution of the difference equation (47.1).

Proof. Let $h^{*}(x, \xi, \mathfrak{F})=\lim _{m \rightarrow \infty} h_{m}(x, \xi, \mathfrak{F}), x \in \mathcal{S}_{\alpha}(\xi)$. For every $\gamma$ greater than $\alpha$ and less than $\beta$ let $X(\gamma)$ be a positive number such that $\boldsymbol{B}_{\gamma(\gamma)}\left[h_{m}(x, \xi, \mathfrak{F})\right]<1$. (Cf. Lemma 31.) The family of functions $\left\{h_{m}(x, \xi, \mathfrak{F})\right\}$, is thus equi-bounded in $\delta_{\gamma}(X(\gamma))$, and therefore, by the well known compactness theorem for bounded families of analytic functions $\left({ }^{15}\right)$, every subsequence of the family contains a partial subsequence convergent in $\delta_{\gamma}(X(\gamma))$, with local uniformity, to a limit function $h(x, \xi, \mathfrak{F})$. Evidently $h(x, \xi, \mathfrak{F})$ is an analytic continuation of $h^{*}(x, \xi, \mathfrak{F})$, and therefore the limit function in $S_{\gamma}(X(\gamma))$ is independent of the particular subsequence. Hence the sequence $\left\{h_{m}(x, \xi, \mathfrak{F})\right\}$ itself converges, with local uniformity, in $\mathcal{S}_{\gamma}(X(\gamma))$, to a limit function $h(x, \xi, \mathfrak{F})$.

Evidently $B_{\gamma X(\gamma)}[h(x, \xi, \mathfrak{F})] \leqq 1$. Also $h(+\infty, \xi, \mathfrak{F})=0$, by Lemma 28 . Hence $h(x, \xi, \mathfrak{F})$ is admissible.

Now it follows from (54.2) that $\sum_{j=1}^{n} a_{k j} h_{m+1}\left(x+\omega_{j}, \xi, \mathfrak{F}\right)=Q_{k} h_{m}(x, \xi, \mathfrak{F})$, from which $\sum_{j=1}^{n} a_{k j} h\left(x+\omega_{j}, \xi, \mathfrak{F}\right)=\boldsymbol{Q}_{k} h(x, \xi, \mathfrak{F})$, which coincides with $(47.1)$ when $h(x)$ is replaced in $(47.1)$ by $h(x, \xi, \mathfrak{F})$.

67. Lemma 33. The set $\mathcal{T}_{k}$ of Theorem 2 is not empty.

Proof. Let $y(x)=\sigma_{k}+h(x, \xi, \mathfrak{F})$, where $h(x, \xi, \mathfrak{F})$ is the function in Lemma 32. (We might, for example, choose all the $C_{8 j m}=0$.) This function $y(x)$ is evidently an element of $\mathcal{T}_{k}$.

68. Notation. Let $V=\max \left\{\left|\omega_{j}\right| \sin (\beta-\alpha) \csc \alpha ; j=2, \cdots, n\right\}$.

69. Lemma 34. If $\xi$ is any non-negative number, and if $x$ is in $\mathcal{S}_{\alpha}(\xi)$, then $x+\omega_{j}$ is in $\mathcal{S}_{\alpha}(\xi+V)(j=2, \cdots, n)$.

Proof. This follows readily from the law of sines, together with the fact

(') Cf. Montel, Leģons sur les familles normales . . , Paris, 1927, p. 21. 
that $\left|\arg \omega_{j}\right| \leqq \beta_{1}$ (cf. $\S 8$ ), and the fact that $\alpha-\beta_{1}=\beta-\alpha$.

70. Lemma 35. There exists a positive number $M_{3}$ such that if $\nu_{t}$ $\left(t=0, \cdots, q_{k}-1\right)$ are any $q_{k}$ complex numbers and if a function $\rho(x, \xi)$ of the form $\sum_{s=1}^{p} \sum_{j_{s}-1}^{j_{s}-1} C_{s j}(x-\xi)^{j_{e} e_{\theta}(x-\xi)}$ is determined by the conditions $D^{t} \rho(\xi+V, \xi)=\nu_{t}\left(t=0,1, \cdots, q_{k}-1\right)$, then $U\left[C_{s j}\right] \leqq M_{3} U\left[\nu_{t}\right]$.

Proof. This is obvious.

71. Lemma 36. There exists a positive number $M_{4}$ such that if $f(x)$ is any function which is in $\mathcal{C}_{\alpha}(\xi)$, then $U\left[D^{t} f(\xi+V) ;\left(t=0,1, \cdots, q_{k}-1\right)\right]$ $\leqq M_{4} B_{\alpha \xi}[f(x)]$.

Proof. This follows at once from the Cauchy integral formula.

72. Lemma 37. There exists a positive number $M_{5}$ such that if $\rho(x, \xi)$ is any function of the form $\sum_{s=1}^{p} \sum_{j=0}^{j_{s}-1} C_{8 j}(x-\xi)^{j} e^{\zeta_{s}(x-\xi)}$, then $B_{\alpha \xi}[\rho(x, \xi)] \leqq M_{5} U\left[C_{8 j}\right]$.

Proof. This follows at once from Lemma 8.

73. LEMma 38. Let $M_{6}=M_{3} M_{5}$. Then if $\rho(x, \xi)$ is any function of the form $\sum_{s=1}^{p} \sum_{j=0}^{j_{s}-1} C_{s j}(x-\xi)^{i} e^{\xi_{\theta}(x-\xi)}$, the inequality $B_{\alpha \xi}[\rho(x, \xi)] \leqq M_{6} U\left[D^{t}\right.$ $\left.\cdot \rho(\xi+V, \xi) ;\left(t=0,1, \cdots, q_{k}-1\right)\right]$ is valid.

Proof. This is a trivial consequence of Lemmas 37 and 35.

74. Lemma 39. Let $H=H(x)$ be an admissible solution of (47.1) such that $H(+\infty)=0$. Then under the transformation $h(x)=H(x)+g(x)$, equation (47.1) is equivalent to the equation

$$
\begin{aligned}
\sum_{j=1}^{n} a_{k j} g\left(x+\omega_{j}\right) & =\sum_{j=1}^{n} E_{k j}(x, H) g\left(x+\omega_{j}\right) \\
+ & \left.\sum_{1} f\left(i_{1}, \cdots, i_{n} ; x ; H\right) g^{i_{1}}\left(x+\omega_{1}\right) \cdots g^{i_{n}}\left(x+\omega_{n}\right)\right)
\end{aligned}
$$

where the coefficients $E_{k j}(x, H)(j=1,2, \cdots, n)$ and the coefficients $f\left(i_{1}, \cdots, i_{n}\right.$; $x ; H)\left(2 \leqq i_{1}+\cdots+i_{n} \leqq d\right)$ are admissible, and where $E_{k j}(+\infty, H)=0$ $(j=1,2, \cdots, n)$.

Moreover, for every positive $\epsilon$ there is a positive $\delta_{3, \epsilon}$, independent of the choice of $H(x)$, such that if $B_{\alpha \xi}[H(x)] \leqq \delta_{3, \epsilon}$, and if $\xi \geqq \xi_{2}$, then $B_{\alpha \xi}\left[E_{k j}(x, H)\right]$ $\leqq B_{\alpha \xi}\left[e_{k j}(x)\right]+\epsilon(j=1,2, \cdots, n)$.

Finally, there exists a positive number $M_{7}$ independent of $H(x)$ such that if $\xi \geqq \xi_{2}$ and if $\boldsymbol{B}_{\alpha \xi}[H(x)] \leqq 1$, then $\boldsymbol{B}_{\alpha \xi}\left[f\left(i_{1}, \cdots, i_{n} ; x ; H\right)\right] \leqq M_{7}\left(2 \leqq i_{1}+\cdots\right.$ $\left.+i_{n} \leqq d\right)$.

Proof. This is obvious.

75. Notation. Let $R_{k H}\left(x ; g_{1}, \cdots, g_{n}\right)$ be the polynomial in indeterminates $g_{1}, \cdots, g_{n}$, with coefficients admissible functions of $x$, defined, in the nota- 
tions of Lemma 39, by the equation

$$
R_{k H}\left(x ; g_{1}, \cdots, g_{n}\right) \equiv \sum_{j=1}^{n} E_{k j}(x, H) g_{j}+\sum_{1} f\left(i_{1}, \cdots, i_{n} ; x ; H\right) g_{1}^{i_{1}} \cdots g_{n}^{i_{n}} .
$$

76. Notation. Let the operator $\boldsymbol{R}_{k H}$ be defined by the following equation, which is to be valid for every function $g(x)$ for which it is meaningful:

$$
\boldsymbol{R}_{k H} g(k)=R_{k H}\left(x ; g\left(x+\omega_{1}\right), \cdots, g\left(x+\omega_{n}\right)\right) .
$$

77. Notation. Let $\epsilon_{1}=\left[6 n M_{8} W(\alpha)\right]^{-1}$, where $M_{8}=1+M_{4} M_{6}$. (Cf. Lemmas $36,38$.

78. Notation. Let $\delta_{4}$ be the minimum of two numbers, the first of which is unity, and the second of which is the value which $\delta_{3, \epsilon}$ assumes when $\epsilon=\epsilon_{1}$. (Cf. Lemma 39.) We note that $\delta_{4}$ is independent of $H$.

79. Notation. Let $\xi_{3}$ be the greatest lower bound of all non-negative real numbers $X$ such that $X \geqq \xi_{2}$ and such that $B_{\alpha X}\left[e_{k j}(x)\right] \leqq \epsilon_{1}(j=1,2, \cdots, n)$.

80. Notation. Let $\xi_{4, H}$ be the greatest lower bound of all non-negative real numbers $X$ such that $X \geqq \xi_{3}$, and $\boldsymbol{B}_{\alpha X}[H(x)] \leqq \delta_{4}$.

81. LemMA 40. If $\xi \geqq \xi_{4, H}$, then

$$
B_{\alpha \xi}\left[E_{k j}(x, H)\right] \leqq\left[3 n M_{8} W(\alpha)\right]^{-1} \quad(j=1,2, \cdots, n)
$$

and

$$
B_{\alpha \xi}\left[f\left(i_{1}, \cdots, i_{n} ; x ; H\right)\right] \leqq M_{7} .
$$

Proof. This follows immediately from Lemma 39.

82. Definition. Let $\mathfrak{F}=\left\{C_{8 j m} ; j=0, \cdots, j_{s}-1 ; s=1, \cdots, p ; m\right.$ $=0,1, \cdots\}$ be an arbitrary sequence of complex numbers. Let $\xi \geqq \xi_{4, H}$. By the sequence $\left\{g_{m}(x ; H ; \xi ; \mathfrak{F})\right\}$ will be meant the sequence of functions defined recursively by the equations

$$
g_{0}(x ; H ; \xi ; \mathfrak{F})=\sum_{s=1}^{p} \sum_{j=0}^{j_{s}-1} C_{s j 0}(x-\xi)^{j} e^{\xi_{*}(x-\xi)}
$$

and

$$
\begin{aligned}
g_{m+1}(x ; H ; \xi ; \mathfrak{F})= & N_{\xi} R_{k H} g_{m}(x ; H ; \xi ; \mathfrak{F}) \\
& +\sum_{s=1}^{p} \sum_{j=0}^{j \delta-1} C_{s j, m+1}(x-\xi)^{j} e^{\xi_{\bullet}(x-\xi)}
\end{aligned}
$$

83. Lemma 41. For every positive number $E$ there exists a positive number $\eta_{1, E}$ independent of $H$ such that if $\xi \geqq \xi_{4, H}$ and $U[\mathfrak{F}] \leqq \eta_{1, E}$, then the sequence $\left\{g_{m}(x ; H ; \xi ; \mathfrak{F})\right\}$ satisfies the inequalities

$$
B_{\alpha \xi}\left[g_{m}(x ; H ; \xi ; \mathfrak{F})\right] \leqq E \quad(m=0,1, \cdots) .
$$


Proof. This follows from exactly the same argument as was used to prove Lemma 27. Inequality (81.1) is to be compared with the analogous inequality (53.1), and it is to be noted that since there is in $R_{k H}\left(x ; g_{1}, \cdots, g_{n}\right)$ no term free of the $g_{j}$ corresponding to the term $\psi_{k}(x)$ in $Q_{k}\left(x ; h_{1}, \cdots, h_{n}\right)$, there is no analogue to the number $\rho_{E}$ of Lemma 27.

84. Lemma 42. There exists a positive number $E_{1}$, independent of $H$, which has the following property:

Let $E$ be any positive number less than or equal to $E_{1}$, let $\mathfrak{F}$ satisfy the condition $U[\mathfrak{F}] \leqq \eta_{1, E}$, let $\xi \geqq \xi_{4, H}$, and let it be given that for each fixed pair $s, j$ the series $\sum_{m=0}^{\infty}\left|C_{s j, m+1}-C_{s j m}\right|$ converges. Then the sequence $\left\{g_{m}(x ; H ; \xi ; \mathfrak{F})\right\}$ converges, with local uniformity, in a region $R$ which contains $\mathcal{S}_{\alpha}(\xi)$, to an admissible solution of the difference equation (74.1).

Proof. This follows from the same arguments as those used to prove Lemmas 28-32.

85. Definition. Let $V$ be the positive number defined in $\$ 68$. Let $\lambda_{t}$ $\left(t=0, \cdots, q_{k}-1\right)$ be any $q_{k}$ complex numbers. Let $\xi \geqq \xi_{4, H}$, and let a sequence of functions $\left\{g_{m}(x ; H ; \xi ; \mathfrak{F})\right\}$ be defined by (82.1) and (82.2), where the $C_{s j m}$ are to be determined by the requirements $D^{t} g_{m}(\xi+V ; H ; \xi, \mathfrak{F})=\lambda_{t}$ $\left(t=0,1, \cdots, q_{k}-1\right)$.

86. Notation. Let $E_{2}$ be the largest positive number such that $M_{8} W(\alpha) b M_{7}\left(2 E_{2}+\cdots+d E_{2}^{d-1}\right) \leqq 1 / 3$. (Cf. $\$ \S 74$ and 77 .) Let $\eta_{2}$ be the value which the $\eta_{1, E}$ of Lemma 41 assumes when $E=E_{1}$. Let $E_{3}$ $=\min \left\{E_{2}, \eta_{2} /\left(M_{3} M_{4}\right)\right\}$.

87. Notation. Let $\delta_{5}$ be the largest positive number such that the equation $X=\delta_{5}+X / 3+b M_{7} M_{8} W(\alpha)\left(X^{2}+\cdots+X^{d}\right)$ has a positive solution $X_{1}$ less than or equal to $E_{3}$.

88. Notation. Let $\eta_{3}=\delta_{5} / M_{6}$.

89. Lemma 43. If $U\left[\lambda_{t}\right] \leqq \eta_{3}$, then the sequence $\left\{g_{m}(x ; H ; \xi ; \mathfrak{F})\right\}$ defined in $\$ 85$ converges, with local uniformity, in a region $R$ which contains $\mathcal{S}_{\alpha}(\xi)$, to an admissible solution $g(x ; H ; \xi ; \mathfrak{F})$ of the difference equation (74.1). Moreover $D^{t} g(\xi+V ; H ; \xi ; \mathfrak{F})=\lambda_{t}\left(t=0,1, \cdots, q_{k}-1\right)$. Also $B_{\alpha \xi}[g(x ; H ; \xi ; \mathfrak{F})] \leqq E_{3}$, and $B_{\alpha \xi}[g(x ; H ; \xi ; \mathfrak{F})]$ approaches zero with $U\left[\lambda_{t}\right]$, uniformly in $H$.

Proof. Let $\rho_{m}(x ; H ; \quad \xi ; \mathfrak{F})=\sum_{s=0}^{p} \sum_{j=0}^{j_{s}-1} C_{s j m}(x-\xi)^{i} e^{\xi_{s}(x-\xi)}$, let $G_{m}$ $=B_{\alpha \xi}\left[g_{m}(x ; H ; \xi ; \mathfrak{F})\right]$, and let $P_{m}=B_{\alpha \xi}\left[\rho_{m}(x ; H ; \xi ; \mathfrak{F})\right]$. Let $\delta_{6, H}$ $=\max \left[B_{\alpha \xi_{4, H}}\left[E_{k j}(x)\right] ; j=1,2, \cdots, n\right\}$. Let $\delta_{7}=M_{6} U\left[\lambda_{t}\right]$. For the next few paragraphs we shall drop the arguments $H, \xi$, and $\mathfrak{F}$.

By Lemma 38 we have $P_{m} \leqq M_{6} U\left[D^{t} \rho_{m}(\xi+V) ;(m=0,1, \cdots)\right]$. But $D^{t} \rho_{0}(\xi+V)=\lambda_{t}\left(t=0,1, \cdots, q_{k}-1\right)$. Hence $P_{0} \leqq \delta_{7}$. Hence $G_{0} \leqq \delta_{7}$.

Now $\rho_{m+1}(x)=g_{m+1}(x)-N_{\xi} R_{k} g_{m}(x)$. Hence $D^{t} \rho_{m+1}(\xi+V)=\lambda_{t}-D^{t} N_{\xi} R_{k} g_{m}$ $\cdot(\xi+V)$. Therefore, using Lemma 36 , we have

$$
U\left[D^{t} \rho_{m+1}(\xi+V)\right] \leqq U\left[\lambda_{t}\right]+M_{4} B_{\alpha \xi}\left[N_{\xi} R_{k} g_{m}(x)\right] .
$$


Consequently, using Lemma 38, we have

$$
P_{m+1} \leqq \delta_{7}+M_{6} M_{4} B_{\alpha \xi}\left[N_{\xi} R_{k} g_{m}(x)\right] .
$$

But $G_{m+1} \leqq B_{\alpha \xi}\left[N_{\xi} R_{k} g_{m}(x)\right]+P_{m+1}$. Hence $G_{m+1} \leqq \delta_{7}+M_{8} B_{\alpha \xi}\left[N_{\xi} R_{k} g_{m}(x)\right]$, where $M_{8}$ is defined in $\S 77$. Therefore, using (81.2), we have

$$
G_{m+1} \leqq \delta_{7}+M_{8} W(\alpha)\left\{n \delta_{6, H} G_{m}+b M_{7}\left(G_{m}^{2}+\cdots+G_{m}^{d}\right)\right\} .
$$

Now $M_{8} W(\alpha) n \delta_{6, H} \leqq 1 / 3$, by (81.1). Hence

$$
G_{m+1} \leqq \delta_{7}+(1 / 3) G_{m}+M_{8} W(\alpha) b M_{7}\left(G_{m}^{2}+\cdots+G_{m}^{d}\right) .
$$

Now the number $X_{1}$ appearing in $\S 87$ is surely greater than $\delta_{5}$, which in turn is not less than $\delta_{7}$. Hence $G_{0} \leqq X_{1}$. But if $G_{m} \leqq X_{1}$, then $G_{m+1} \leqq \delta_{5}+(1 / 3) X_{1}$ $+M_{8} W(\alpha) b M_{7}\left(X_{1}^{2}+\cdots+X_{1}^{d}\right)=X_{1}$. Hence $G_{m} \leqq X_{1}(m=0,1, \cdots)$. Hence, using (89.1), we see readily that $P_{m} \leqq X_{1}(m=0,1, \cdots)$. Thus $G_{m} \leqq E_{3}$ and $P_{m} \leqq E_{3}(m=0,1, \cdots)$. Hence by Lemmas 35 and 36 ,

$$
U[\mathfrak{F}] \leqq M_{4} M_{3} E_{3} \leqq \eta_{2}
$$

Now let $\Delta g_{m}(x)=g_{m+1}(x)-g_{m}(x)$, let $\Delta \rho_{m}(x)=\rho_{m+1}(x)-\rho_{m}(x)$, let $Q_{m}$ $=B_{\alpha \xi}\left[\Delta g_{m}(x)\right]$, and let $S_{m}=B_{\alpha \xi}\left[\Delta \rho_{m}(x)\right]$. Then $\Delta g_{m}(x)=v_{m}(x)+\Delta \rho_{m}(x)$, where $v_{m}(x)=N_{\xi}\left(\boldsymbol{R}_{k} g_{m}(x)-\boldsymbol{R}_{k} g_{m-1}(x)\right)$. Hence

$$
\begin{aligned}
D^{t}\left(\Delta \rho_{m}(\xi+V)\right) & =D^{t}\left(\Delta g_{m}(\xi+V)\right)-D^{t} v_{m}(\xi+V) \\
& =0-D^{t} v_{m}(\xi+V) .
\end{aligned}
$$

Hence $U\left[D^{t} \Delta \rho_{m}(\xi+V)\right] \leqq M_{4} B_{\alpha \xi}\left[v_{m}(x)\right]$. Therefore $S_{m} \leqq M_{6} M_{4} B_{\alpha \xi}\left[v_{m}(x)\right]$. But $Q_{m} \leqq B_{\alpha \xi}\left[v_{m}(x)\right]+S_{m}$. Hence $Q_{m} \leqq M_{8} B_{\alpha \xi}\left[v_{m}(x)\right]$. Therefore

$$
Q_{m} \leqq M_{8} W(\alpha)\left\{\delta_{6, H} n Q_{m-1}+b M_{7} Q_{m-1}\left(2 E_{3}+3 E_{3}^{2}+\cdots+d E_{3}^{d-1}\right)\right\} .
$$

Hence, from $\S 86$ and (81.1), it follows that $Q_{m} \leqq(2 / 3) Q_{m-1}$. Hence $Q_{m}$ $\leqq(2 / 3)^{m} Q_{0} \leqq(2 / 3)^{m}\left(2 E_{3}\right)$. Now $S_{m} \leqq M_{6} M_{4} B_{\alpha \xi}\left[v_{m}(x)\right] \leqq M_{6} M_{4} W(\alpha)\left\{\delta_{6, H} n Q_{m-1}\right.$ $\left.+b M_{7} Q_{m-1}\left(2 E_{3}+3 E_{3}^{2}+\cdots+d E_{3}^{d-1}\right)\right\} \leqq(2 / 3) Q_{m-1}$. Hence $S_{m} \leqq(2 / 3)^{m}\left(2 E_{3}\right)$. Hence $\sum_{m=0}^{\infty} S_{m}$ converges. But $\Delta \rho_{m}(x)=\sum_{s=1}^{p} \quad \sum_{j=0}^{j_{s}-1}\left(C_{s j, m+1}-C_{s j m}\right)$ $\cdot(x-\xi)^{j} e^{\zeta_{s}(x-\xi)}$. Hence $U\left[C_{s j, m+1}-C_{s j m}\right] \leqq M_{3} M_{4} S_{m}$, by Lemmas 35 and 36 . Thus, for each $s, j$ the series $\sum_{m=0}^{\infty}\left|C_{8 j, m+1}-C_{8 j m}\right|$ converges.

This last statement, taken in conjunction with (89.4), allows us to apply Lemma 42 , and to conclude therefore that the sequence $\left\{g_{m}(x ; H ; \xi ; \mathfrak{F})\right\}$ converges with local uniformity to an admissible solution $g(x ; H ; \xi ; \mathfrak{F})$ of $(74.1)$; evidently $g(x ; H ; \xi ; \mathfrak{F})$ will be analytic in $\mathcal{S}_{\alpha}(\xi)$, and $B_{\alpha \xi}[g(x ; H ; \xi ; \mathfrak{F})] \leqq E_{3}$. Also $D^{t} g(\xi+V ; H ; \xi ; \mathfrak{F})=\lambda_{t}\left(t=0,1, \cdots, q_{k}-1\right)$.

Now if $\epsilon^{*}$ is any positive number, let $\delta^{*}$ be the largest positive number such that the equation $X=\delta^{*}+(1 / 3) X+M_{8} W(\alpha) b M_{7}\left(X^{2}+\cdots+X^{d}\right)$ has a positive solution $X^{*}$ less than or equal to $\epsilon^{*}$. If $\delta_{7} \leqq \delta^{*}$, then $\delta_{7} \leqq X^{*}$, whence 
$G_{0} \leqq X^{*}$. But by equation (89.3) we have, if $\delta_{7} \leqq \delta^{*}$, the inequality $G_{m+1} \leqq \delta^{*}$ $+(1 / 3) G_{m}+M_{8} W(\alpha) b M_{7}\left(G_{m}^{2}+\cdots+G_{m}^{d}\right)$, and therefore, by complete induction, $G_{m} \leqq X^{*} \leqq \epsilon^{*}$. Thus $B_{\alpha \xi}[g(x ; H ; \xi ; \mathfrak{F})] \leqq \epsilon^{*}$, provided $\delta_{7} \leqq \delta^{*}$. This establishes the last statement of the lemma.

90. LEMmA 44. If $U\left[\lambda_{t}\right] \leqq \eta_{3}$ and $\xi \geqq \xi_{4, H}$, then there exists at most one admissible solution $g(x)$ of (74.1) such that $B_{\alpha \xi}[g(x)] \leqq E_{3}$ and such that $D^{t} g(\xi+V)$ $=\lambda_{t}\left(t=0,1, \cdots, q_{k}-1\right)$.

Proof. Assume $g_{1}(x)$ and $g_{2}(x)$ are two admissible solutions of (74.1) such that $B_{\alpha \xi}\left[g_{i}(x)\right] \leqq E_{3}$ and $D^{t} g_{i}(\xi+V)=\lambda_{t}\left(t=0,1, \cdots, q_{k}-1 ; i=1,2\right)$.

Let $u(x)=g_{2}(x)-g_{1}(x)$.

Then $\sum_{j=1}^{n} a_{k j} u\left(x+\omega_{j}\right)=\boldsymbol{R}_{k} g_{2}(x)-\boldsymbol{R}_{k} g_{1}(x)$. Hence, by Theorem 1 , we have $u(x)=v(x, \xi)+\rho(x, \xi)$ where $v(x, \xi)=N_{\xi}\left[\boldsymbol{R}_{k} g_{2}(x)-\boldsymbol{R}_{k} g_{1}(x)\right]$, and where $\rho(x, \xi)$ is a function of the form $\sum_{s=1}^{p} \sum_{j=0}^{y_{\xi}-1} C_{s j}(x-\xi)^{i} e^{\zeta_{s}(x-\xi)}$. Let $u^{*}$ $=B_{\alpha \xi}[u(x)], v^{*}=B_{\alpha \xi}[v(x, \xi)]$ and $\rho^{*}=B_{\alpha \xi}[\rho(x, \xi)]$. Now $D^{t} \rho(\xi+V, \xi)$ $=D^{t} u(\xi+V, \xi)-D^{t} v(\xi+V, \xi)=0-D^{t} v(\xi+V, \xi)$. Hence $U\left[D^{t} \rho(\xi+V, \xi)\right]$ $=U\left[D^{t} v(\xi+V, \xi)\right] \leqq M_{4} v^{*}$. Hence $\rho^{*} \leqq M_{6} M_{4} v^{*}$. But $u^{*} \leqq v^{*}+\rho^{*}$. Hence $u^{*} \leqq\left(1+M_{4} M_{6}\right) v^{*}=M_{8} v^{*}$. Noting $\S \S 77$ and 81 we see that $u^{*} \leqq M_{8} W(\alpha)$ $\cdot\left\{2 n \epsilon_{1} u^{*}+b M_{7} u^{*}\left(2 E_{3}+3 E_{3}^{2}+\cdots+d E_{3}^{d-1}\right)\right\}$. But, noting $\$ \$ 77$ and 86 , we see that $2 n \epsilon_{1} M_{8} W(\alpha) \leqq 1 / 3$ and that $M_{8} W(\alpha) b M_{7}\left(2 E_{3}+\cdots+d E_{3}^{d-1}\right) \leqq 1 / 3$. Hence $u^{*} \leqq(2 / 3) u^{*}$. Hence $u^{*}=0$. Hence $g_{1}(x) \equiv g_{2}(x)$.

91. LEMMA 45. Let $y_{0}(x)$ be any admissible solution of (42.1) such that $y_{0}(+\infty)=\sigma_{k}$. Let $H(x)=y_{0}(x)-\sigma_{k}$, and let $\xi_{4, H}$ be the number defined, in terms of $H(x)$, in $\$ \$ 77-80$. Let $E_{3}, \eta_{3}$ be the numbers, independent of $H$, defined in $\$ \S 86$ and 88 . Let $\xi \geqq \xi_{4, H}$. Let $\lambda_{t}\left(t=0,1, \cdots, q_{k}-1\right)$ be any complex numbers such that $U\left[\lambda_{t}\right] \leqq \eta_{3}$. Then there exists one, and only one, admissible solution $y(x)$ of (42.1) such that $B_{\alpha \xi}\left[y(x)-y_{0}(x)\right] \leqq E_{3}$, such that $y(+\infty)=\sigma_{k}$, and such that $D^{t} y(\xi+V)=D^{t} y_{0}(\xi+V)+\lambda_{t}\left(t=0,1, \cdots, q_{k}-1\right)$. Moreover there exists a positive-valued function $\eta_{4}(\epsilon) \leqq \eta_{3}$, independent of $H(x)$ and of $\xi$, such that $B_{\alpha \xi}\left[y(x)-y_{0}(x)\right]<\epsilon$ whenever $U\left[\lambda_{t}\right] \leqq \eta_{4}(\epsilon)$.

Proof. Let $g(x ; H ; \xi ; \mathfrak{F})$ be the function appearing in the statement of Lemma 43. Let $y(x)=H(x)+\sigma_{k}+g(x ; H ; \xi ; \mathfrak{F})$. Then $y(x)$ satisfies all the required conditions.

Suppose on the other hand that there are two functions $y(x)$ satisfying all the stated conditions. Let one be called $y_{1}(x)$, the other $y_{2}(x)$. Let $g_{i}(x)=y_{i}(x)$ $-H(x)-\sigma_{k}(i=1,2)$. Then $g_{i}(x)$ is a solution of (74.1) which satisfies the conditions $\boldsymbol{B}_{\alpha \xi}\left[g_{i}(x)\right] \leqq E_{3}, D^{t} g_{i}(\xi+V)=\lambda_{t}\left(t=0,1, \cdots, q_{k}-1\right)(i=1,2)$. Hence, by Lemma $44, g_{1}(x) \equiv g_{2}(x)$. Hence $y_{1}(x) \equiv y_{2}(x)$.

The last statement in the lemma follows from the last statement in Lemma 43.

92. Notation. Let $\rho_{k}$ be equal to one-half the distance from the number $\sigma_{k}$ to the set of all finite roots $y_{1}$ other than $\sigma_{k}$ of the equation $\Lambda\left(+\infty, y_{1}, \sigma_{k}\right.$, 
$\left.\sigma_{k}, \cdots, \sigma_{k}\right)=0$.

93. LeMma 46. There exists a positive number $\xi_{5} \geqq \xi_{3}$ and a positive number $\delta_{8}<\rho_{k}$ such that

(a) For each set of complex numbers $x, y_{2}, \cdots, y_{n}$ with $x$ in $\mathcal{S}_{\alpha}\left(\xi_{5}\right)$ and with $\left|y_{2}-\sigma_{k}\right| \leqq \delta_{8}$ there is exactly one complex number $y_{1}$ satisfying the equation $\Lambda\left(x, y_{1}, y_{2}, \cdots, y_{n}\right)=0$ and lying in the circle $\left|y_{1}-\sigma_{k}\right|<\rho_{k}$, and

(b) The $y_{1}$ of (a) is given by the formula

$$
y_{1}=P_{k}\left(x, y_{2}, \cdots, y_{n}\right) \equiv \frac{1}{2 \pi i} \int_{\mathcal{C}} \frac{\zeta \partial \Lambda\left(x, \zeta, y_{2}, \cdots, y_{n}\right) / \partial \zeta}{\Lambda\left(x, \zeta, y_{2}, \cdots, y_{n}\right)} d \zeta
$$

where $\mathcal{C}$ is the positively sensed circle $\left[\zeta-\sigma_{k} \mid=\rho_{k}\right.$, and

(c) $\Lambda\left(x, \zeta, y_{2}, \cdots, y_{n}\right)$ is bounded away from zero provided the relations $\left|\zeta-\sigma_{k}\right|=\rho_{k}, x \in \mathcal{S}_{\alpha}\left(\xi_{5}\right)$, and $\left|y_{j}-\sigma_{k}\right| \leqq \delta_{8}(j=2,3, \cdots, n)$ are all satisfied.

Proof. This is an immediate consequence of the fact that $\Lambda(+\infty$, $\left.\sigma_{k}, \cdots, \sigma_{k}\right)=0$, while $\partial \Lambda\left(+\infty, \sigma_{k}, \cdots, \sigma_{k}\right) / \partial y_{1} \neq 0$.

94. Lemma 47. Let $\xi \geqq \xi_{5}$. Let $y(x)$ be a solution of (42.1) which is in $\mathcal{C}_{\alpha}(\xi)$ and satisfies the inequality $B_{\alpha \xi}\left[y(x)-\sigma_{k}\right] \leqq \delta_{8}$. Then $y(x) \equiv P_{k}\left(x, y\left(x+\omega_{2}\right), \cdots\right.$, $\left.y\left(x+\omega_{n}\right)\right)$ throughout $\mathcal{S}_{\alpha}(\xi)$.

Proof. This is a corollary of Lemma 46.

95. Lemma 48. Let $\xi \geqq \xi_{5}+V$. Let $y(x)$ be a solution of (42.1) which is analytic in $\mathcal{S}_{\alpha}(\xi)$ and satisfies the inequality $B_{\alpha \xi}\left[y(x)-\sigma_{k}\right] \leqq \delta_{8}$. Then there is an analytic continuation $Y(x)$ of $y(x)$ throughout the sector $\mathcal{S}_{\alpha}(\xi-V)$, which is given by

$$
Y(x) \equiv P_{k}\left(x, y\left(x+\omega_{2}\right), \cdots, y\left(x+\omega_{n}\right)\right) \quad \text { when } \quad x \in \mathcal{S}_{\alpha}(\xi-V) .
$$

Proof. When $x \in \mathcal{S}_{\alpha}(\xi-V)$, then $x+\omega_{j} \in \mathcal{S}_{\alpha}(\xi)(j=2, \cdots, n)$. (Cf. Lemma 34.) Hence the right-hand member of (95.1) is analytic throughout $\mathcal{S}_{\alpha}(\xi-V)$. Since this right-hand member coincides with $y(x)$ when $x \in S_{\alpha}(\xi)$ (cf. Lemma 47 ), it follows that $Y(x)$ as given by (95.1) is an analytic continuation of $y(x)$ throughout $\delta_{\alpha}(\xi-V)$.

96. Notation. Let $\xi_{6}=\xi_{5}+V$.

97. LEMMA 49. For every positive $\epsilon$ there exists a positive $\delta_{9}(\epsilon)$ such that if $x \in \mathcal{S}_{\alpha}\left(\xi_{6}\right)$ and $y_{2}^{\prime}, \ldots, y_{n}^{\prime} ; y_{2}^{\prime \prime}, \ldots, y_{n}^{\prime \prime}$ are complex numbers such that $\left|y_{j}^{\prime}-\sigma_{k}\right| \leqq \delta_{8}, \quad\left|y_{j}^{\prime \prime}-\sigma_{k}\right| \leqq \delta_{8}, \quad$ and $\quad\left|y_{j}^{\prime}-y_{j}^{\prime \prime}\right| \leqq \delta_{9}(\epsilon) \quad(j=2, \cdots, n)$, then $\left|P_{k}\left(x, y_{2}^{\prime}, \cdots, y_{n}^{\prime}\right)-P\left(x, y_{2}^{\prime \prime}, \cdots, y_{n}^{\prime \prime}\right)\right| \leqq \epsilon$.

Proof. This is an obvious corollary of Lemma 46.

98. Notation. Let $\delta_{10}=\min \left\{(1 / 2) \delta_{8},(1 / 2) \delta_{4}\right\}$. (Cf. §78.)

99. Notation. Let $E_{4}=\min \left\{E_{3}, \delta_{9}\left(E_{3}\right),(1 / 2) \delta_{8}\right\}$.

100. Notation. Let $\eta_{5}=\eta_{4}\left(E_{4}\right)$ where $\eta_{4}(\epsilon)$ is the function defined in 
Lemma 45.

101. LEMMA 50. Let $y_{0}(x)$ be any admissible solution of (42.1) such that $y_{0}(+\infty)=\sigma_{k}$. Let $\eta_{5}$ be the number defined in $\$ 100$. Let $\xi_{7, y_{0}(x)}$ be the greatest lower bound of all real numbers $X$ such that $X \geqq \xi_{6}$ and such that $B_{\alpha X}\left[y_{0}(x)-\sigma_{k}\right]$ $\leqq \delta_{10}$. Let $\xi \geqq V+\xi_{7, y_{0}(x)}$ and let $\lambda_{t}\left(t=0,1, \cdots, q_{k}-1\right)$ be any set of $q_{k}$ complex numbers such that $U\left[\lambda_{t}\right] \leqq \eta_{5}$. Then

(a) There exists one and only one admissible solution $y(x)$ of (42.1) such that $y(+\infty)=\sigma_{k}$, such that $B_{\alpha \xi}\left[y(x)-y_{0}(x)\right] \leqq E_{4}$, and such that $D^{t} y(\xi)$ $=D^{t} y_{0}(\xi)+\lambda_{t}\left(t=0,1, \cdots, q_{k}-1\right)$. Moreover, 45.)

(b) If $\epsilon \leqq E_{4}$, then $B_{\alpha \xi}\left[y(x)-y_{\theta}(x)\right] \leqq \epsilon$ whenever $U\left[\lambda_{t}\right] \leqq \eta_{4}(\epsilon)$. (Cf. Lemma

Proof. Let $H=H(x)=y_{0}(x)-\sigma_{k}$. We have $\xi-V \geqq \xi_{7, y_{0}} \geqq \xi_{4, H}$. Hence by Lemma 45 there is an admissible solution $y(x)$ of (42.1) such that $\boldsymbol{B}_{\alpha, \xi-V}\left[y(x)-y_{0}(x)\right] \leqq E_{4}$, such that $D^{t} y(\xi-V+V)=D^{t} y_{0}(\xi-V+V)+\lambda_{t}$, $\left(t=0,1, \cdots, q_{k}-1\right)$, and such that $y(+\infty)=\sigma_{k}$. A fortiori we have $B_{\alpha \xi}\left[y(x)-y_{0}(x)\right] \leqq E_{4}$. This proves the existence of at least one $y(x)$ of the required sort.

Suppose now that $y(x)$ is any function of the required sort. Evidently $B_{\alpha \xi}\left[y(x)-\sigma_{k}\right] \leqq B_{\alpha \xi}\left[y(x)-y_{0}(x)\right]+B_{\alpha \xi}\left[y_{0}(x)-\sigma_{k}\right] \leqq E_{4}+\delta_{10} \leqq \delta_{8}$. Hence, by Lemma 48, $y(x)$ has an analytic continuation $Y(x)$ throughout $\delta_{\alpha}(\xi-V)$ given by

$$
Y(x)=P_{k}\left(x, y\left(x+\omega_{2}\right), \cdots, y\left(x+\omega_{n}\right)\right),
$$

and also $y_{0}(x)$ has an analytic continuation $Y_{0}(x)$ throughout $\mathcal{S}_{\alpha}(\xi-V)$ given by

$$
Y_{0}(x)=P_{k}\left(x, y_{0}\left(x+\omega_{2}\right), \cdots, y_{0}\left(x+\omega_{n}\right)\right) .
$$

Now if $x \in S_{\alpha}(\xi-V)$, then $x+\omega_{j} \in S_{\alpha}(\xi) \quad(j=2, \cdots, n)$. Hence, if $x \in S_{\alpha}(\xi-V)$, then $\left|y\left(x+\omega_{j}\right)-\sigma_{k}\right| \leqq\left|y\left(x+\omega_{j}\right)-y_{0}\left(x+\omega_{j}\right)\right|+\mid\left(y_{0}\left(x+\omega_{j}\right)-\sigma_{k} \mid\right.$ $\leqq E_{4}+\delta_{10} \leqq(1 / 2) \delta_{8}+(1 / 2) \delta_{8}=\delta_{8}$. Also $\left|y_{0}\left(x+\omega_{j}\right)-\sigma_{k}\right| \leqq(1 / 2) \delta_{8}$ and $\mid y\left(x+\omega_{j}\right)$ $-y_{0}\left(x+\omega_{j}\right) \mid \leqq E_{4} \leqq \delta_{9}\left(E_{3}\right)$. Hence, by Lemma 49 and equations (101.1) and (101.2), we have $\left|Y(x)-Y_{0}(x)\right| \leqq E_{3}$ if $x \in \mathcal{S}_{\alpha}(\xi-V)$. Thus $Y_{0}(x), Y(x)$ are admissible solutions of (42.1) with $B_{\alpha, \xi-V}\left[Y(x)-Y_{0}(x)\right] \leqq E_{3}$ and $D^{t} Y(\xi-V$ $+V)=D^{t} Y_{0}(\xi-V+V)+\lambda_{t}(t=0,1,2, \cdots)$. Since $\xi-V \geqq \xi_{4, H}$, it follows from Lemma 45 that $Y(x)$ is unique. Hence $y(x)$ is unique.

Statement (b) follows readily from the last statement of Lemma 45.

Part VII. A metric for the space $\mathcal{T}_{k}$.

102. Notation. Let $y(x)$ be any function in $\mathcal{T}_{k}$. By $\mu[y(x)]$ will be meant the greatest lower bound of all positive numbers $X$ such that $X \geqq \xi_{6}$ (cf. $\S \S 96,93$ ) and such that $B_{\alpha X}\left[y(x)-\sigma_{k}\right]<\delta_{10}$ (cf. \$98). (In other notation, $\mu[y(x)]$ $=\xi_{7, y(x)}$, as defined in $\$ 101$.)

103. Definition. Let $y_{1}(x), y_{2}(x)$ be any two functions in $\mathcal{\tau}_{k}$. Let $X^{\prime}$ 
$=\max \left\{\mu\left[y_{1}(x)\right], \mu\left[y_{2}(x)\right]\right\}$. By the step from $y_{1}(x)$ to $y_{2}(x)$, written $S\left[y_{1}(x), y_{2}(x)\right]$, will be meant $\left|\mu\left[y_{2}(x)\right]-\mu\left[y_{1}(x)\right]\right|+B_{\alpha X^{\prime}}\left[y_{2}(x)-y_{1}(x)\right]$.

104. Definition. Let $y_{1}(x), y_{2}(x)$ be any two functions in $\mathcal{T}_{k}$. Then by the distance from $y_{1}(x)$ to $y_{2}(x)$, written $d\left[y_{1}(x), y_{2}(x)\right]$, is meant the greatest lower bound of all numbers $\sum_{m=0}^{N} S\left[f_{m}(x), f_{m+1}(x)\right]$ where $N$ is an arbitrary positive integer and the $f_{m}(x)(m=0, \cdots, N+1)$ are any functions in $\mathcal{\sigma}_{k}$ such that $f_{0}(x) \equiv y_{1}(x)$ and $f_{N+1}(x) \equiv y_{2}(x)$.

105. Lemma 51. The function $d\left[y_{1}(x), y_{2}(x)\right]$ is a metric; that is to say

(a) $\boldsymbol{d}\left[y_{1}(x), y_{2}(x)\right]=\boldsymbol{d}\left[y_{2}(x), y_{1}(x)\right]$,

(b) $\boldsymbol{d}\left[y_{1}(x), y_{2}(x)\right] \geqq 0$,

(c) $d\left[y_{1}(x), y_{2}(x)\right]=0$ if and only if $y_{1}(x) \equiv y_{2}(x)$,

(d) $d\left[y_{1}(x), y_{2}(x)\right] \leqq d\left[y_{1}(x), y_{3}(x)\right]+d\left[y_{3}(x), y_{2}(x)\right]$.

Proof. (a), (b), (d), and the first part of (c) are obvious. Also it is easy to see that if $\boldsymbol{d}\left[y_{1}(x), y_{2}(x)\right]<\epsilon$, then $B_{\alpha, x^{*}+\epsilon}\left[y_{2}(x)-y_{1}(x)\right]<\epsilon$, where $X^{*}$ $=\mu\left[y_{1}(x)\right]$. This obviously implies the second part of (c).

106. Lemma 52. Let $y_{0}(x)$ be any function belonging to $\mathcal{G}_{k}$. Let $X$ be any non-negative real number such that $y_{0}(x)$ is in $\mathcal{C}_{\alpha}(X)$. Then if $\left\{y_{m}(x)\right\}$ $(m=1,2, \cdots)$ is any sequence of functions belonging to $\mathcal{T}_{k}$ such that the sequence $\left\{B_{\alpha X}\left[y_{m}(x)-y_{0}(x)\right]\right\}$ is a null sequence, then the sequence $\left\{\boldsymbol{d}\left[y_{m}(x)\right.\right.$, $\left.\left.y_{0}(x)\right]\right\}$ is a null sequence.

Proof. By Lemma 48 we have the result that if $\xi$ is any real number such that $\xi \geqq \xi_{5}+V$, then any element $y(x)$ of $\mathcal{T}_{k}$ can be continued analytically throughout the region $\mathcal{S}_{\alpha}(\xi-V)$ by means of the equation

$$
y(x)=P_{k}\left(x, y\left(x+\omega_{2}\right), \cdots, y\left(x+\omega_{n}\right)\right),
$$

provided $y(x)$ is analytic in $S_{\alpha}(\xi)$ and $B_{\alpha \xi}\left[y(x)-\sigma_{k}\right] \leqq \delta_{8}$. Similarly $y_{0}(x)$ can be continued analytically throughout the region $\mathcal{S}_{\alpha}(\xi-V)$ by means of the equation

$$
y_{0}(x)=P_{k}\left(x, y_{0}\left(x+\omega_{2}\right), \cdots, y_{0}\left(x+\omega_{n}\right)\right),
$$

provided $\xi \geqq \xi_{5}+V$ and provided $y_{0}(x)$ is analytic in $\mathcal{S}_{\alpha}(\xi)$ with $B_{\alpha \xi}\left[y_{0}(x)-\sigma_{k}\right]$ $\leqq \delta_{8}$.

Suppose first that $X<\mu\left[y_{0}(x)\right]=G$. Then the sequence $\left\{B_{\alpha} G^{\prime}\left[y_{0}(x)\right.\right.$ $\left.\left.-y_{m}(x)\right]\right\}$ is a null sequence for some $G^{\prime}$ less than $G$ (namely, for $G^{\prime}=X$ ).

Suppose on the other hand that $X \geqq G$. Then $X \geqq \xi_{6}=\xi_{5}+V$. Also $B_{\alpha X}\left[y_{0}(x)-\sigma_{k}\right] \leqq \delta_{10} \leqq(1 / 2) \delta_{8}<\delta_{8}$. Hence equation (106.2) can be applied with $\xi=X$. Also, there exists a positive $m_{1}$ such that if $m \geqq m_{1}$, then $B_{\alpha X}\left[y_{m}(x)-\sigma_{k}\right]$, which is not greater than $B_{\alpha X}\left[y_{m}(x)-y_{0}(x)\right]+B_{\alpha X}\left[y_{0}(x)\right.$ $\left.-\sigma_{k}\right]$, is less than $(1 / 2) \delta_{8}+(1 / 2) \delta_{8}=\delta_{8}$. Hence, if $m \geqq m_{1}$, equation (106.1) can be applied, with $\xi=X$ and $y(x)=y_{m}(x)$. Then by Lemma 49 the sequence $\left\{\boldsymbol{B}_{\alpha, X-V}\left[y_{m}(x)-y_{0}(x)\right]\right\}\left(m \geqq m_{1}\right)$ is a null sequence. 
Suppose now that $X-V<G$. Then the sequence $B_{\alpha G^{\prime}}\left[y_{m}(x)-y(x)\right]$ $\left(m \geqq m_{1}\right)$ is a null sequence for some $G^{\prime}$ less than $G$ (namely, for $G^{\prime}=X-V$ ).

If, on the other hand, $X-V \geqq G$, then the discussion of the preceding paragraphs, with $X$ replaced by $X-V$, can be applied to the sequence $\left\{y_{m}(x) ; m \geqq m_{1}\right\}$ to show that for some $m_{2} \geqq m_{1}$ the sequence $\left\{B_{\alpha, X-2 V}\left[y_{m}(x)\right.\right.$ $\left.\left.-y_{0}(x)\right]\right\}\left(m \geqq m_{2}\right)$ is a null sequence. A finite number of repetitions of this argument leads to the result that there exists a positive integer $m^{*}$ such that if $m \geqq m^{*}$, the function $y_{m}(x)$ can be continued analytically over a sector $S_{\alpha}(\xi)$ with $\xi<\mu\left[y_{0}(x)\right]$, and for some $G^{\prime}$ less than $G$ the sequence $\left\{B_{\alpha} G^{\prime}\left[y_{m}(x)\right.\right.$ $\left.\left.-y_{0}(x)\right] ; m \geqq m^{*}\right\}$ is a null sequence.

Now if $\xi$ is any number greater than $G$, we have $B_{\alpha \xi}\left[y_{0}(x)-\sigma_{k}\right]<\delta_{10}$ (by definition of $\mu\left[y_{0}(x)\right]$ ). Hence if $m$ is large, $B_{\alpha \xi}\left[y_{m}(x)-\sigma_{k}\right]<\delta_{10}$. Hence, since $\xi>\xi_{6}$, we have $\mu\left[y_{m}(x)\right] \leqq \xi$ if $m$ is large. Thus

$$
\limsup _{m \rightarrow \infty} \mu\left[y_{m}(x)\right] \leqq G \text {. }
$$

Let $F=\lim$ inf $\mu\left[y_{m}(x)\right]$. Suppose that $F<G$. Let $\mathfrak{M}$ be an infinite sequence of positive integers such that

$$
F=\lim _{m \rightarrow \infty, m \in \mathfrak{M}} \mu\left[y_{m}(x)\right] .
$$

Let $H$ be a number which is less than $G$ but which is greater than $G^{\prime}$ and greater than $F$. Then if $m$ is large, $\mu\left[y_{m}(x)\right]<H(m \in \mathfrak{M})$. This implies that if $m$ is large, $B_{\alpha H}\left[y_{m}(x)-\sigma_{k}\right]<\delta_{10}(m \in \mathfrak{M})$. But the sequence $\left\{\boldsymbol{B}_{\alpha H}\left[y_{m}(x)\right.\right.$ $\left.\left.-y_{0}(x)\right] ; m \geqq m^{*}\right\}$ is a null sequence. Hence $\boldsymbol{B}_{\alpha H}\left[y_{0}(x)-\sigma_{k}\right] \leqq \delta_{10}$. Hence if $\xi$ is any number greater than $H, B_{\alpha \xi}\left[y_{0}(x)-\sigma_{k}\right]<\delta_{10}$. (Cf. Lemma 6.) This last inequality, together with the fact that $H>\xi_{0}$ (since $F \geqq \xi_{6}$ ), leads to the conclusion that $\mu\left[y_{0}(x)\right] \leqq H$. This contradiction to the definition of $G$ forces us to reject the possibility that $F<G$.

Hence $F \geqq G$. This, together with (106.3), shows that $\lim \mu\left[y_{m}(x)\right]$ exists and equals $\mu\left[y_{0}(x)\right]$. This, taken in conjunction with the fact that $\lim B_{\alpha G}\left[y_{m}(x)-y_{0}(x)\right]$ is zero, shows that $\left\{\delta\left[y_{0}(x), y_{m}(x)\right]\right\}$ is a null sequence, whence $\left\{\boldsymbol{d}\left[y_{0}(x), y_{m}(x)\right]\right\}$ is a null sequence.

107. Lемма 53. The set $\mathcal{G}_{k}$ is a connected space, in the metric $\boldsymbol{d}\left[y_{1}, y_{2}\right]$.

Proof. Let $\delta^{*}=\min \left\{(1 / 2) \delta_{4},(1 / 2) E_{3}\right\}$ (cf. $\$ \$ 78$ and 86 ). Let $y_{1}(x), y_{2}(x)$ be any two functions belonging to $\mathcal{T}_{k}$. Let $\xi$ be any number such that $\xi \geqq \xi_{3}$, such that $B_{\alpha \xi}\left[y_{1}(x)-\sigma_{k}\right] \leqq(1 / 2) \delta_{4}$, such that $B_{\alpha \xi}\left[y_{2}(x)-y_{1}(x)\right] \leqq E_{3}$, and such that $\left|D^{t}\left[y_{2}(\xi+V)-y_{1}(\xi+V)\right]\right| \leqq \eta_{4}\left(\delta^{*}\right)$ (cf. Lemma 45) $(t=0,1, \cdots$, $q_{k}-1$ ). (Obviously such a $\xi$ exists since every derivative of $y_{2}(x)-y_{1}(x)$ tends to zero as $x$ becomes positively infinite.)

Let $\lambda_{t}=D^{t} y_{2}(\xi+V)-D^{t} y_{1}(\xi+V) \quad\left(t=0,1, \cdots, q_{k}-1\right)$. Then $U\left[\lambda_{t}\right]$ $\leqq \eta_{4}\left(\delta^{*}\right) \leqq \eta_{3}$. Let $y(x, r)(0 \leqq r \leqq 1)$ be that function, which by Lemma 45 is unique, which belongs to $\mathcal{T}_{k}$ and satisfies the relations $B_{\alpha \xi}\left[y(x, r)-y_{1}(x)\right]$ 
$\leqq E_{3}, D^{t} y(\xi+V, r)=D^{t} y_{1}(\xi+V)+r \lambda_{t}\left(t=0,1, \cdots, q_{k}-1\right)$. Evidently $y(x, 0)$ $\equiv y_{1}(x)$ and $y(x, 1) \equiv y_{2}(x)$. Now $U\left[r \lambda_{t}\right] \leqq \eta_{4}\left(\delta^{*}\right)$. Hence $B_{\alpha \xi}\left[y(x, r)-y_{1}(x)\right]$ $\leqq \delta^{*}$. This implies that if $0 \leqq r \leqq 1$ and $0 \leqq s \leqq 1$, then $B_{\alpha \xi}[y(x, r)-y(x, s)] \leqq E_{3}$. Also it implies that $B_{\alpha \xi}\left[y(x, r)-y_{1}(x)\right] \leqq(1 / 2) \delta_{4}$. But $B_{\alpha \xi}\left[y_{1}(x)-\sigma_{k}\right] \leqq(1 / 2) \delta_{4}$. Hence $B_{\alpha \xi}\left[y(x, r)-\sigma_{k}\right] \leqq \delta_{4}$. Hence if $r$ is any number in $[0,1]$ and if $\left\{r_{m}\right\}$ is a sequence of numbers in $[0,1]$ converging to $r$, it follows that since $\left\{U\left[D^{t} y\left(\xi+V, r_{m}\right)-D^{t} y(\xi+V, r)\right]\right\}$ is evidently a null sequence, the last statement of Lemma 45 can be applied, with $y_{0}(x)$ replaced by $y(x, r)$, to show that $B_{\alpha \xi}\left[y\left(x, r_{m}\right)-y(x, r)\right]$ is a null sequence. Hence by Lemma 52 the sequence $\left\{\boldsymbol{d}\left[y\left(x, r_{m}\right), y(x, r)\right]\right\}$ is a null sequence. Hence the continuum of functions $\{y(x, r) ; 0 \leqq r \leqq 1\}$ is a continuous curve in the space $\mathcal{T}_{k}$. Hence $\mathcal{T}_{k}$ is a connected space.

\section{Lemma 54. $\mathcal{T}_{k}$ is separable.}

Proof. Let $\mathscr{W}_{k}$ be the subset of $\mathcal{T}_{k}$ consisting of all functions $y(x)$ belonging to $\mathcal{T}_{k}$ and having the property that there exists an integer $N$ (depending upon $y(x)$ ) such that the numbers $D^{t} y(N)$ are all rational-complex numbers $\left(t=0,1, \cdots, q_{k}-1\right)$, such that $N \geqq \mu[y(x)]+V$, and such that $B_{\alpha N}\left[y(x)-\sigma_{k}\right] \leqq(1 / 2) E_{4}$. Now there exists for each choice of $N$, and each choice of rational-complex numbers $D^{t} y(N)$, at most one function $y(x)$ with the properties described. For if $y_{0}(x)$ is one such, and $y(x)$ is another, then $\boldsymbol{B}_{\alpha N}\left[y(x)-y_{0}(x)\right] \leqq E_{4}$, and therefore Lemma 50 can be applied with $\lambda_{t}=0$ $\left(t=0,1, \cdots, q_{k}-1\right)$ to show that $y(x) \equiv y_{0}(x)$. Hence $\mathscr{W}_{k}$ is countable.

Now if $y_{0}(x)$ is any function belonging to $\mathcal{T}_{k}$, let a positive integer $N$ be chosen, greater than $\mu\left[y_{0}(x)\right]+V$, and sufficiently large so that $B_{\alpha N}\left[y_{0}(x)-\sigma_{k}\right]<(1 / 2) E_{4}$. Let $\left\{y_{m}(x)\right\} \quad(m=1,2, \cdots)$ be a sequence of functions belonging to $\mathcal{T}_{k}$, such that $B_{\alpha N}\left[y_{m}(x)-y_{0}(x)\right] \leqq E_{4}$, and such that $\left\{\boldsymbol{U}\left[D^{t} y_{m}(N)-D^{t} y_{0}(N)\right]\right\}$ is a null sequence, and such that $D^{t} y_{m}(N)$ is rational $\left(t=0,1, \cdots, q_{k}-1 ; m=1,2, \cdots\right)$. Then $\left\{B_{\alpha N}\left[y_{m}(x)-y_{0}(x)\right]\right\}$ is a null sequence (by Lemma 50b), and therefore, by Lemma 52, $\left\{\boldsymbol{d}\left[y_{m}(x), y_{0}(x)\right]\right\}$ is a null sequence. But $\boldsymbol{d}\left[y_{m}(x), y_{0}(x)\right] \geqq \mid \mu\left[y_{m}(x)\right]$ $-\mu\left[y_{0}(x)\right] \mid$ and therefore $\mu\left[y_{m}(x)\right]+V<N$ if $m$ is large. Also, since $\left\{B_{\alpha N}\left[y_{m}(x)-y_{0}(x)\right]\right\}$ is a null sequence, it follows that if $m$ is large, $B_{\alpha N}\left[y_{m}(x)-\sigma_{k}\right]<E_{4} / 2$. Thus if $m$ is large, $y_{m}(x) \in W_{k}$. Since $d\left[y_{m}(x), y_{0}(x)\right]$ is a null sequence, $\mathscr{W}_{k}$ is dense in $\mathcal{T}_{k}$.

Thus $\mathcal{T}_{k}$ is separable.

109. Lemma 55. $\mathcal{T}_{k}$ is locally homeomorphic with $q_{k}$-dimensional complex Euclidean space.

Proof. Let $y_{0}(x)$ belong to $\mathcal{T}_{k}$. Let $X=\mu\left[y_{0}(x)\right]$. If $\left\{y_{m}(x)\right\}$ is any sequence of functions in $\mathcal{G}_{k}$ such that $\left\{B_{\alpha, X+V}\left[y_{m}(x)-y_{0}(x)\right]\right\}$ is a null sequence, then $\left\{d\left[y_{m}(x), y_{0}(x)\right]\right\}$ is a null sequence, by Lemma 52. Hence $\left\{\mu\left[y_{m}(x)\right]\right.$ $\left.-\mu\left[y_{0}(x)\right]\right\}$ is a null sequence. Hence if $m$ is large, $\mu\left[y_{m}(x)\right]<X+V$. Thus 
there exists a positive number $E_{5}$, which we shall take less than or equal to $(1 / 2) E_{3}$, such that if $y(x)$ is in $\mathcal{G}_{k}$ and if $B_{\alpha, X+V}\left[y(x)-y_{0}(x)\right]<E_{5}$, then $\mu[y(x)]<X+V .\left(E_{5}\right.$ is, at least at first sight, dependent upon $y_{0}(x)$.)

Now $B_{\alpha, X+V}\left[y_{0}(x)-\sigma_{k}\right] \leqq \delta_{10} \leqq(1 / 2) \delta_{4}$.

Let $\eta_{6}=\eta_{4}\left(E_{5}\right)$. Let $\varepsilon$ be the set consisting of all $q_{k}$-tuples of complex numbers $\left\{\lambda_{t} ; t=0,1, \cdots, q_{k}-1\right\}$ such that $U\left[\lambda_{t}\right]<\eta_{6}$. Let $\mathcal{N}$ be the set in $\tau_{k}$ consisting of all functions in $\mathcal{G}_{k}$ for which $B_{\alpha, X+V}\left[y(x)-y_{0}(x)\right]<E_{5}$, and for which the set $\left.\left\{D^{t}\left[y(X+2 V)-y_{0}(X+2 V)\right] ; t=0,1, \cdots, q_{k}-1\right)\right\}$ is in $\varepsilon$. Let that mapping from $\mathcal{N}$ to $\varepsilon$ be defined which maps each element $y(x)$ of $\mathcal{N}$ into the set $\left\{D^{t}\left[y(X+2 V)-y_{0}(X+2 V)\right]\right\}$ belonging to $\varepsilon$. By Lemma 45 the mapping is one-to-one.

If $y(x)$ is in $\mathcal{N}$, then $B_{\alpha, X+V}\left[y(x)-y_{0}(x)\right]<E_{5}$. Hence $\mu[y(x)]<X+V$. This implies that for every $y(x)$ in $\mathcal{N}$ we have

$$
X+V>\xi_{4, y}(x)-\sigma_{k} \text {. }
$$

Now $\mathcal{N}$ is an open set in $\mathcal{T}_{k}$ : For if $y(x)$ is any element of $\mathcal{N}$, and if $\left\{y_{m}(x)\right\}$ is any sequence of elements of $\mathcal{T}_{k}$ such that $\left\{\boldsymbol{d}\left[y_{m}(x), y(x)\right]\right\}$ is a null sequence, then $\left\{\mu\left[y_{m}(x)\right]-\mu[y(x)]\right\}$ is a null sequence, so that if $m$ is large, $\mu\left[y_{m}(x)\right]<X+V$. Consequently, since $\mu[y(x)]<X+V$ and $\left\{d\left[y_{m}(x), y(x)\right]\right\}$ is a null sequence, it follows that $\left\{B_{\alpha, X+V}\left[y_{m}(x)-y(x)\right]\right\}$ is a null sequence. Hence if $m$ is large, $\left\{\boldsymbol{D}^{t}\left[y_{m}(X+2 V)-y_{0}(X+2 V)\right]\right\}$ is in $\mathcal{E}$. Also, since $\left\{B_{\alpha, X+V}\left[y_{m}(x)-y(x)\right]\right\}$ is a null sequence and since $B_{\alpha, X+V}\left[y(x)-y_{0}(x)\right]<E_{5}$, we see that if $m$ is large, then $\boldsymbol{B}_{\alpha, X+V}\left[y_{m}(x)-y_{0}(x)\right]<E_{5}$. Hence if $m$ is large, $y_{m}(x)$ is in $\mathcal{N}$. Hence $\mathcal{N}$ is open.

Now the mapping from $\mathcal{N}$ to $\mathcal{E}$ is continuous. For if $\left\{y_{m}(x)\right\}$ is a sequence of functions in $\mathcal{N}$ converging to a function $y(x)$ in $\mathcal{N}$, then, since $\mu[f(x)]$ $<X+V$ for every $f(x)$ in $\mathcal{N}$, we conclude that $\left\{B_{\alpha, X+V}\left[y_{m}(x)-y(x)\right]\right\}$ is a null sequence. Hence $\left\{\boldsymbol{U}\left[\boldsymbol{D}^{t}\left[y_{m}(X+2 V), y(X+2 V)\right]\right]\right\}$ is a null sequence. Hence the point of $\mathcal{E}$ corresponding to $y_{m}(x)$ tends, as $m$ becomes infinite, to the point of $\varepsilon$ corresponding to $y(x)$.

Finally, the mapping from $\mathcal{E}$ to $\mathcal{N}$ is continuous. For if $\left\{\left\{\lambda_{m t}\right.\right.$; $\left.\left.t=0,1, \cdots, q_{k}-1\right\} ; m=1,2, \cdots\right\}$ is a sequence of elements of $\varepsilon$ tending to the element $\left\{\lambda_{t} ; t=0,1, \cdots, q_{k}-1\right\}$ of $\mathcal{E}$ and if $y(x), y_{m}(x)$ are the elements of $\mathcal{N}$ corresponding to $\left\{\lambda_{t}\right\},\left\{\lambda_{m t}\right\}$ respectively, then $\left\{U\left[D^{t}\left[y_{m}(X+2 V)\right.\right.\right.$ $-y(X+2 V)]]\}$ is a null sequence, and therefore we see, using Lemma 45 with $y_{0}(x)$ replaced by $y(x)$, and $y(x)$ replaced by $y_{m}(x)$, and with $\xi=X+V$ (note (109.1)), that $\left\{B_{\alpha, X+V}\left[y_{m}(x)-y(x)\right]\right\}$ is a null sequence. Hence, by Lemma $52,\left\{d\left[y_{m}(x), y(x)\right]\right\}$ is a null sequence. Thus the mapping from $\varepsilon$ to $\mathcal{N}$ is continuous.

110. Lemma 56. Theorem 2 is valid.

Proof. This follows immediately from Lemmas 21, 50a, 51, 53, 54, and 55 . 111. REMARK. With very slight changes it is possible to introduce in a 
natural way a metric for the whole set $\mathcal{T}$, in terms of which the sets $\mathcal{T}_{k}$ become precisely the connected components of $\mathcal{T}$, and as before the $\mathcal{T}_{k}$ become $q_{k}$-dimensional manifolds.

Part VIII. Examples.

112. EXAMPLE I.

$$
\begin{aligned}
& \left(1+x^{-1}\right) y^{2}(x) y(x+1)-17 y^{2}(x+1) \\
& +15\left(1+x^{-2} \log x\right) y(x+1)+y(x)-x e^{-x}=0 .
\end{aligned}
$$

Here $n=2$, and $\Lambda\left(x, y_{1}, y_{2}\right)=\left(1+x^{-1}\right) y_{1}^{2} y_{2}-17 y_{2}^{2}+15\left(1+x^{-2} \log x\right) y_{2}+y_{1}-x e^{-x}$, so that

$$
\Lambda\left(+\infty, y_{1}, y_{2}\right)=y_{1}^{2} y_{2}-17 y_{2}^{2}+15 y_{2}+y_{1}
$$

whence

$$
J(\sigma)=\sigma^{3}-17 \sigma^{2}+16 \sigma,
$$

and therefore $c=3$, and $\sigma_{1}=0, \sigma_{2}=1, \sigma_{3}=16$. The corresponding exponential polynomials are $f_{1}(z) \equiv 1+15 e^{z}, f_{2}(z) \equiv 3-18 e^{z}, f_{3}(z) \equiv 513-273 e^{z}$. It is readily seen, therefore, on the basis of Theorem 2 , that if, for instance, $\beta=\pi / 36$, then the parameter numbers are given by $q_{1}=10, q_{2}=7$, and $q_{3}=0$. Similarly if $\beta=\pi / 12$, then $q_{1}=4, q_{2}=3$, and $q_{3}=0$. If $\beta=\pi / 6$, then $q_{1}=2, q_{2}=1$, and $q_{3}=0$. If $\beta=\pi / 3$, then $q_{1}=0, q_{2}=1$, and $q_{3}=0$.

113. EXAMPLE II.

$$
16 y^{2}(x+1)-8 y(x) y(x+1)+y^{2}(x)+4 y(x+1)-2 y(x)=0 .
$$

This example is similar to Example I. However, it has the very special property that it is possible to determine the set of all analytic solutions (admissible or not) explicitly, and to see exactly how the admissible solutions fit into the totality of all solutions. It is possible also to see that the uniqueness of the admissible solution satisfying assigned initial conditions, as described in Theorem 2 , is a uniqueness which is strictly local; in general there will be in the set of all admissible solutions of (113.1), with limit $\sigma_{k}$ at $+\infty$, exactly two functions satisfying a given set of $q_{k}$ assigned initial conditions.

We have $n=2$, and $\Lambda\left(x, y_{1}, y_{2}\right)=\Lambda\left(+\infty, y_{1}, y_{2}\right)=16 y_{2}^{2}-8 y_{1} y_{2}+y_{1}^{2}+4 y_{2}$ $-2 y_{1}$. Hence $J(\sigma)=9 \sigma^{2}+2 \sigma$. Hence $c=2$, and $\sigma_{1}=0$, while $\sigma_{2}=-2 / 9$. For the auxiliary exponential polynomials we have $f_{1}(z) \equiv-2+4 e^{z}, f_{2}(z)$ $\equiv\left(1+2 e^{z}\right)(-2 / 3)$. Thus, on the basis of Theorem 2 , if, for example, $\pi / 2$ $\geqq \beta>\operatorname{arc} \cot (\pi / \log 2)$, then it is easy to see that $q_{1}=1$ and $q_{2}=0$, while if $\operatorname{arc} \cot (\pi / \log 2) \geqq \beta>\operatorname{arc} \cot (2 \pi / \log 2)$, then $q_{1}=1$ and $q_{2}=2$, and if arc $\cot (2 \pi / \log 2) \geqq \beta>\operatorname{arc} \cot (3 \pi / \log 2)$, then $q_{1}=3$ and $q_{2}=2$.

Now (113.1) can be solved explicitly by the following device. We note that (113.1) is equivalent to 
(113.2)

$$
y(x)=[4 y(x+1)-y(x)]^{2}+[4 y(x+1)-y(x)] .
$$

If in (113.2), multiplied by 4 , we replace $x$ by $x+1$, and if we subtract (113.2) from this resulting equation, we get

$$
\begin{aligned}
4 y(x+1)-y(x)= & 4[4 y(x+2)-y(x+1)]^{2} \\
& +4[4 y(x+2)-y(x+1)] \\
& -[4 y(x+1)-y(x)]^{2} \\
& -[4 y(x+1)-y(x)] .
\end{aligned}
$$

If we set $v(x)=4 y(x+1)-y(x)$, we see that (113.3) can be written

$$
v(x)=4 v^{2}(x+1)+4 v(x+1)-v^{2}(x)-v(x),
$$

whence

$$
[v(x)+1]^{2}=[2 v(x+1)+1]^{2},
$$

so that either

$$
v(x)+1=2 v(x+1)+1
$$

or

$$
v(x)+1=-2 v(x+1)-1 .
$$

The totality of analytic solutions of (113.6a) is

$$
v(x)=C_{1}(x) 2^{-x},
$$

where $C_{1}(x)$ is an arbitrary analytic function of period 1. From (113.7a) we we have $4 y(x+1)-y(x)=C_{1}(x) 2^{-x}$, whence

$$
y(x)=C_{1}(x) 2^{-x}+C_{2}(x) 4^{-x},
$$

where $C_{2}(x)$ is an analytic function of period 1. If (113.8a) is substituted into (113.2), it is seen at once that $y(x)$ as given by (113.8a) is a solution of (113.2) if and only if $C_{2}(x)=C_{1}^{2}(x)$. Thus (113.6a) leads to the set of solutions

$$
y(x)=C_{1}(x) 2^{-x}+C_{1}^{2}(x) 4^{-x},
$$

where $C_{1}(x)$ is an arbitrary analytic function of period 1 .

Similarly (113.6b) leads to the set of solutions

$$
y(x)=(-2 / 9)+(-1 / 3) e^{\pi i x} C_{3}(x) 2^{-x}+e^{2 \pi i x} C_{3}^{2}(x) 4^{-x},
$$

where $C_{3}(x)$ is an arbitrary analytic function of period 1 . Thus the totality of analytic solutions of (113.1) is given by the union of the families of functions (113.9a) and (113.9b). Consequently it is easy to see, by examination of (113.9a) and (113.9b), that when $\pi / 2 \geqq \beta>\operatorname{arc} \cot (\pi / \log 2)$ the set $\tau_{1}$ is the set 


$$
y(x)=C 2^{-x}+C^{2} 4^{-x},
$$

where $C$ is any arbitrary constant, while $\mathcal{T}_{2}$ is the set consisting of the unique function $y(x) \equiv-2 / 9$. When $\operatorname{arc} \cot (\pi / \log 2) \geqq \beta>\operatorname{arc} \cot (2 \pi / \log 2), \mathcal{V}_{1}$ is the set (113.10) while $\mathcal{T}_{2}$ is the set

$$
\begin{aligned}
y(x)= & (-2 / 9)+(-1 / 3)\left(K_{1} e^{\pi i x}+K_{2} e^{-\pi i x}\right) 2^{-x} \\
& +\left(K_{1}^{2} e^{2 \pi i x}+2 K_{1} K_{2}+K_{2}^{2} e^{-2 \pi i x}\right) 4^{-x},
\end{aligned}
$$

where $K_{1}$ and $K_{2}$ are arbitrary constants. When $\operatorname{arc} \cot (2 \pi / \log 2) \geqq \beta$ $>\operatorname{arc} \cot (3 \pi / \log 2), \mathcal{T}_{1}$ is the set

$$
\begin{aligned}
y(x)= & \left(L_{1}+L_{2} e^{2 \pi i x}+L_{3} e^{-2 \pi i x}\right) 2^{-x} \\
& +\left(L_{1}+L_{2} e^{2 \pi i x}+L_{3} e^{-2 \pi i x}\right)^{2} 4^{-x},
\end{aligned}
$$

where $L_{1}, L_{2}$, and $L_{3}$ are arbitrary constants, and $\mathcal{T}_{2}$ is the set (113.11).

To verify the assertion made at the beginning of this example, that the uniqueness is only local, let us consider the case of $\tau_{1}$ when $\beta=\pi / 2$. If $\xi$ is any positive number and $I$ is any complex number different from $(-1 / 4)$ there are clearly two functions $y(x)$ in the set (113.10) such that $y(\xi)=I$. Similar remarks apply in this example to all those cases for which the number of parameters is different from zero.

Columbia University, NEW YoRK, N. Y. 